Article

\title{
Scopularides Revisited: Molecular Networking Guided Exploration of Lipodepsipeptides in Australian Marine Fish Gastrointestinal Tract-Derived Fungi
}

\author{
Ahmed H. Elbanna $\left.{ }^{1}{ }^{(}\right)$, Zeinab G. Khali1 ${ }^{1}\left(\mathbb{D}\right.$, Paul V. Bernhardt ${ }^{2}$ and Robert J. Capon ${ }^{1, *(\mathbb{C}}$ \\ 1 Institute for Molecular Bioscience, The University of Queensland, St Lucia, QLD 4072, Australia \\ 2 School of chemistry and Molecular Bioscience, The University of Queensland, St Lucia, QLD 4072, Australia \\ * Correspondence: r.capon@uq.edu.au; Tel.: +61-7-3346-2979
}

Received: 31 July 2019; Accepted: 14 August 2019; Published: 16 August 2019

\begin{abstract}
Chemical analysis of a cultivation of an Australian Mugil mullet gastrointestinal tract (GIT) derived fungus, Scopulariopsis sp. CMB-F458, yielded the known lipodepsipeptides scopularides A (1) and B (2). A comparative global natural product social (GNPS) molecular networking analysis of $\times 63$ co-isolated fungi, detected two additional fungi producing new scopularides, with Beauveria sp. CMB-F585 yielding scopularides C-G (3-7) and Scopulariopsis sp. CMB-F115 yielding scopularide $\mathrm{H}$ (8). Structures inclusive of absolute configurations were assigned by detailed spectroscopic and $\mathrm{C}_{3}$ Marfey's analysis, together with X-ray analyses of $\mathbf{3}$ and $\mathbf{8}$, and biosynthetic considerations. Scopularides A-H (1-8) did not exhibit significant growth inhibitory activity against a selection of Gram positive (+ve) and negative (-ve) bacteria, a fungus, or a panel of three human carcinoma cell lines.
\end{abstract}

Keywords: Scopulariopsis sp.; Beauveria sp.; lipodepsipeptides; scopularides; GNPS; marine fungal biodiscovery

\section{Introduction}

As part of our ongoing investigation into secondary metabolites from Australian marine-derived fungi, we speculated that selected bottom feeding fish species may act as natural myco-accumulators, and as such could be a readily accessible source of marine-derived fungi. Using three Mugil mullet acquired from a local fish market, we assembled a library of $\sim 500$ chemically-distinct gastrointestinal tract (GIT)-derived fungi. In a preliminary validation of the potential of this resource, we reported on an unprecedented class of hydrazine containing furano Schiff bases, the prolinimines, from Trichoderma sp. CMB-F563 [1]. Building on this achievement, we now describe an investigation into scopularide lipodepsipeptides, literature accounts of which are limited to scopularides A-B (1-2) from the marine sponge-derived fungus Scopulariopsis brevicaulis NCPF-2177 [2]. This current study was prompted by a fortuitous re-isolation of scopularides A-B (1-2) from the Mugil mullet GIT-derived Scopulariopsis sp. CMB-F458. Using 1 and 2 as authentic standards, a comparative global natural product social (GNPS) molecular networking [3] analysis of extracts obtained from $\times 63$ co-isolated GIT-derived fungi, enabled the detection, and subsequent scaled-up cultivation, isolation and identification of the new scopularides C-G (3-7) from Beauveria sp. CMB-F585, and the new scopularide H (8) from Scopulariopsis sp. CMB-F115. Structures were assigned to 3-8 (Figure 1) on the basis of detailed spectroscopic and chemical analysis, as summarised below. 

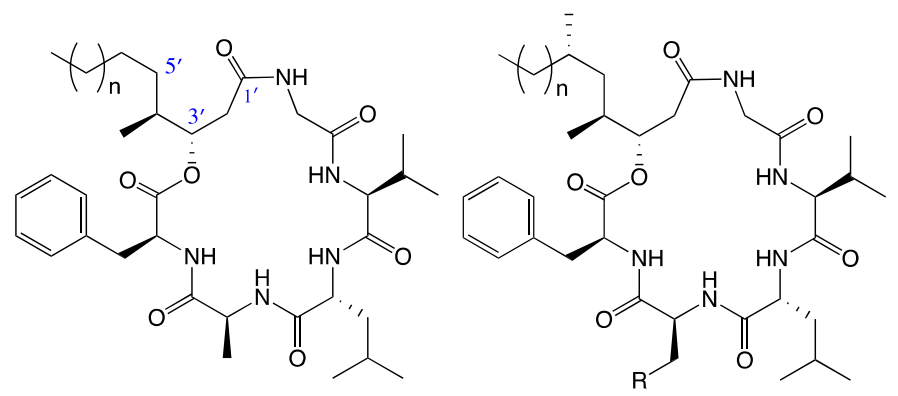

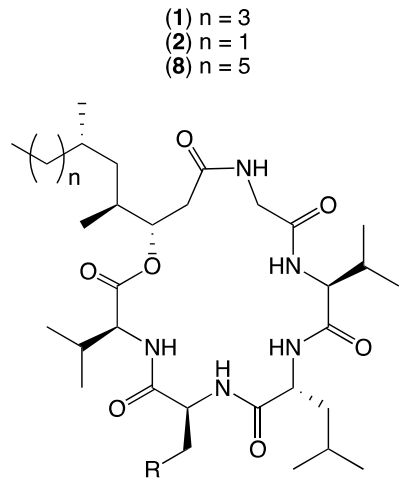

(4) $n=5, R=H$ (7) $n=5, R=M e$

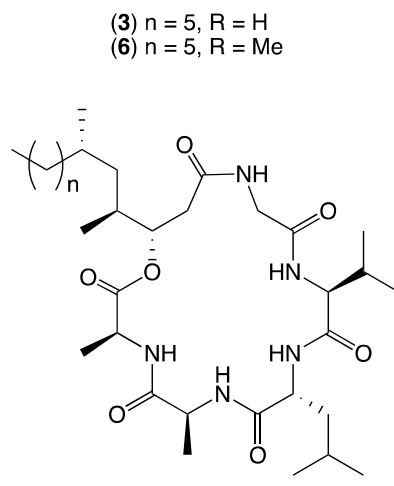

(5) $n=5$

Figure 1. Scopularides A-H (1-8) from Mugil mullet gastrointestinal tract (GIT)-derived fungi.

\section{Results and Discussion}

The EtOAc extract from a M1S solid phase $(20 \times$ agar plate $)$ cultivation of the Mugil mullet GIT-derived fungus Scopulariopsis sp. CMB-F458 was subjected to sequential solvent partitioning followed by reversed-phase HPLC to yield scopularides A-B (1-2). Planar structures for 1-2 were independently assigned by detailed spectroscopic analysis, with amino acid residues inclusive of absolute configurations assigned by $C_{3}$ Marfey's analysis (Figures S46 and S47) [4]. The complete structures for 1-2 were confirmed by 1D NMR (methanol- $d_{4}$ ) in comparison to literature data (Tables S1 and S3) [2]. Armed with authentic standards of 1 and 2, a comparative GNPS analysis of extracts obtained from cultivations of $\times 63$ co-isolated fungi detected a "scopularide" cluster incorporating nodes from Scopulariopsis sp. CMB-F458 and two other fungal strains, Beauveria sp. CMB-F585 and Scopulariopsis sp. CMB-F115 (Figure 2).

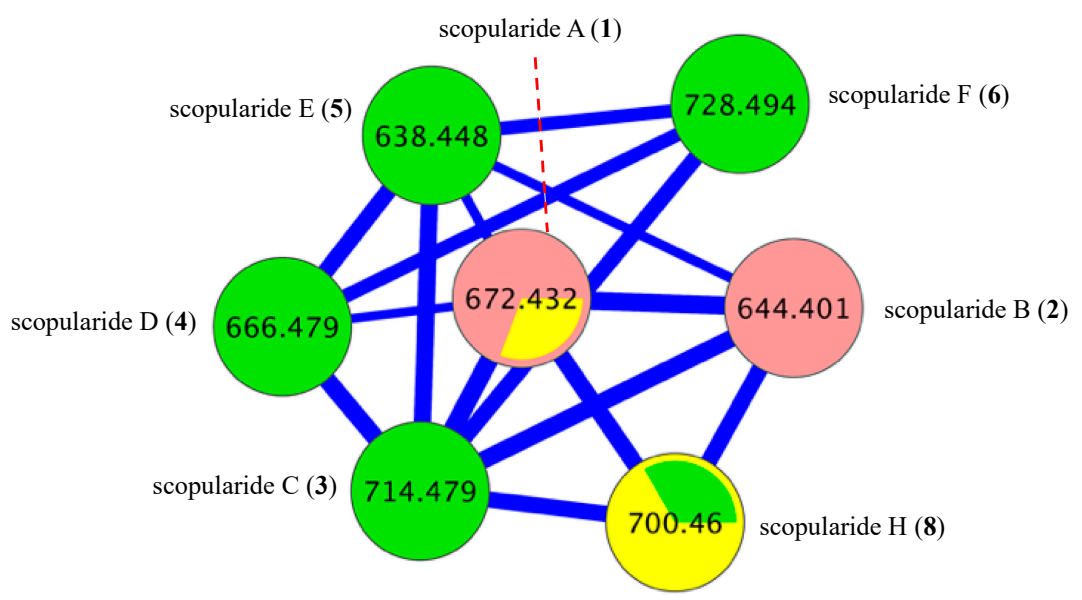

Figure 2. Global natural product social (GNPS) molecular networking cluster for scopularides detected in a comparative analysis of $\times 63$ Mugil mullet GIT-derived fungal extracts. CMB-F458 pink nodes, CMB-F585 green nodes, and CMB-F115 yellow nodes. 
GNPS analysis of the EtOAc extract obtained from a YES solid phase $(80 \times$ agar plate) cultivation of Beauveria sp. CMB-F585 detected the presence of four prominent new scopularides C-F (3-6), together with trace amounts of the new scopularides G-H (7-8) and known scopularide A (1) (Figure 3). Solvent extraction and partitioning of the EtOAc extract, followed by reversed-phase HPLC, yielded five new scopularides 3-7. HRESI(+)MS analysis of scopularide $C$ (3) revealed a molecular formula $\left(\mathrm{C}_{39} \mathrm{H}_{63} \mathrm{~N}_{5} \mathrm{O}_{7}\right)$ consistent with a higher homologue $\left(+\mathrm{C}_{3} \mathrm{H}_{6}\right)$ of scopularide A (1). A C Marfey's analysis (Figure 4) supported by diagnostic of MS/MS fragmentations (Figure 5) and 1D and 2D NMR (DMSO- $d_{6}$ ) (Table 1 and Table S5, Figure 6 and Figures S14-S18) established the amino acid sequence L-Phe ${ }^{1}-\mathrm{L}-\mathrm{Ala}^{2}-\mathrm{D}-\mathrm{Leu}^{3}-\mathrm{L}-\mathrm{Val}^{4}-\mathrm{Gly}^{5}$. The complete structure for scopularide C (3), including absolute configuration about the 3S,4S,6S-3-hydroxyl-4,6-dimethyllauric acid (HDMLA) lipid residue, was confirmed by single crystal X-ray analysis (Figure 7 and Figure S55).

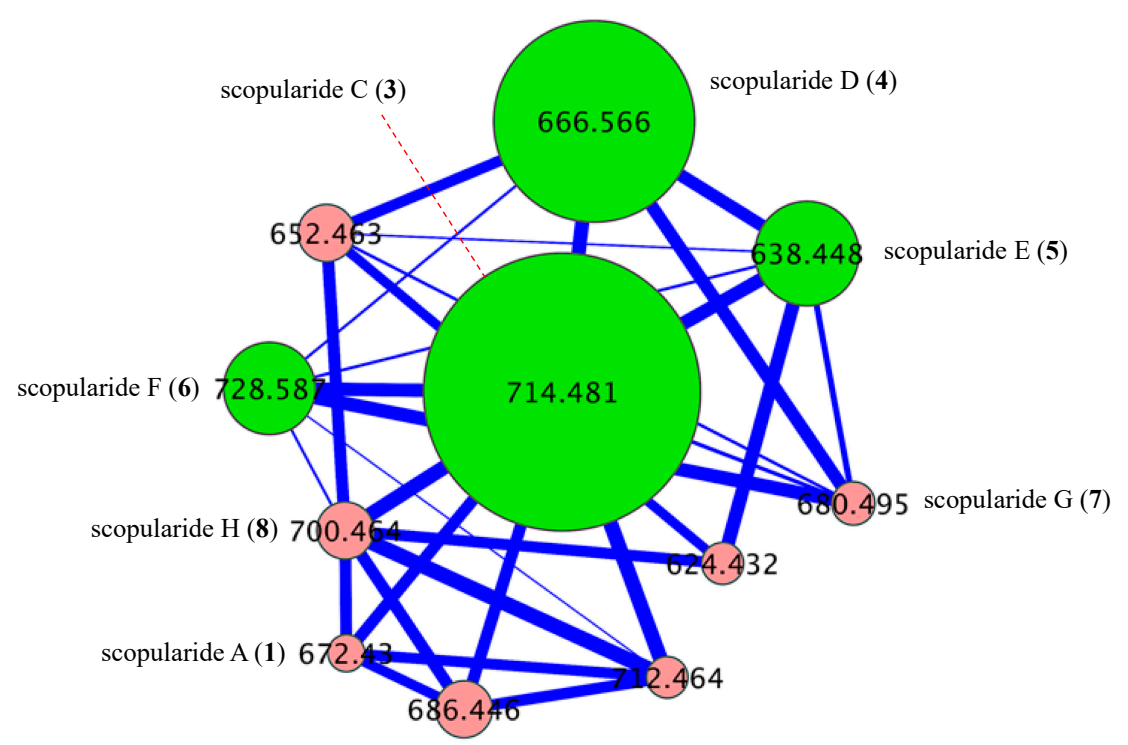

Figure 3. GNPS cluster for scopularides detected in a YES solid phase cultivation of Beauveria sp. CMB-F585. Green nodes are scopularides C-F (3-6), and pink nodes are minor analogues.
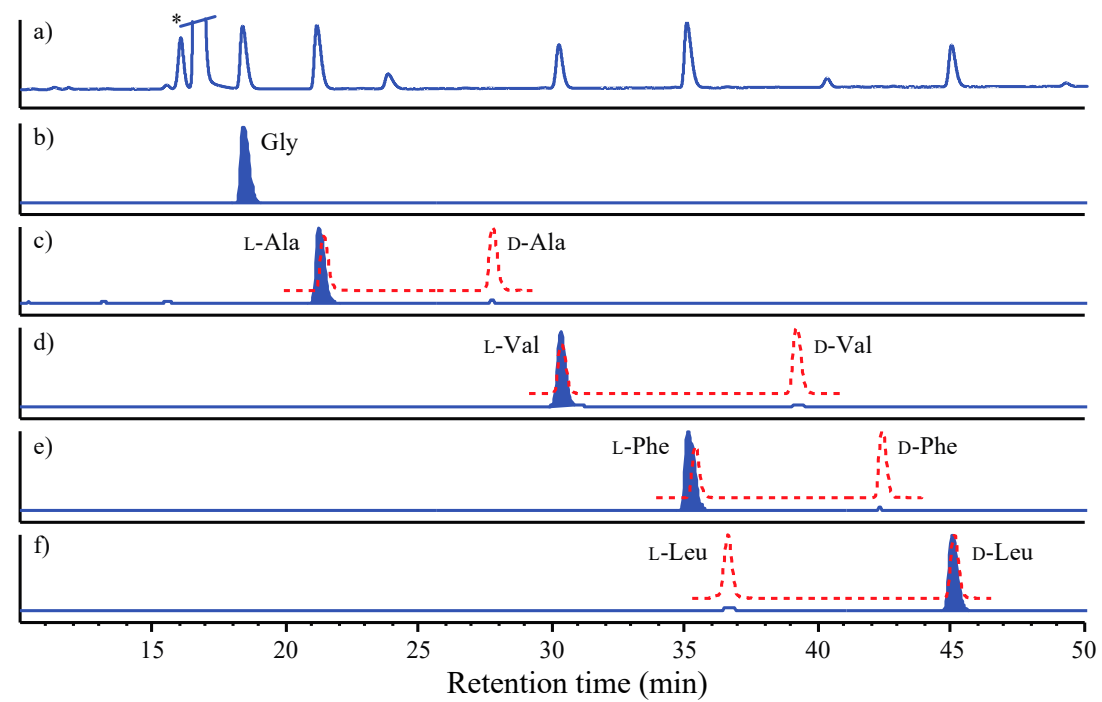

Figure 4. $C_{3}$ Marfey's analysis of scopularide C (3). (a) $C_{3}$ HPLC-DAD (340 nm) chromatogram of L-FDAA amino acid derivatives of acid hydrolysate of an aliquot $(50 \mu \mathrm{g})$ of 3 . (b-f) HPLC-MS single ion extraction (SIE) chromatograms for L-FDAA derivatives of authentic standards (red broken lines) and the acid hydrolysate of 3 (blue shaded peaks). (b) Gly (SIE $m / z$ 328), (c) L-Ala (SIE $m / z$ 342), (d) L-Val (SIE $m / z$ 370), (e) L-Phe (SIE $m / z$ 418) and (f) D-Leu (SIE $m / z$ 384). * Excess Marfey's reagent. 


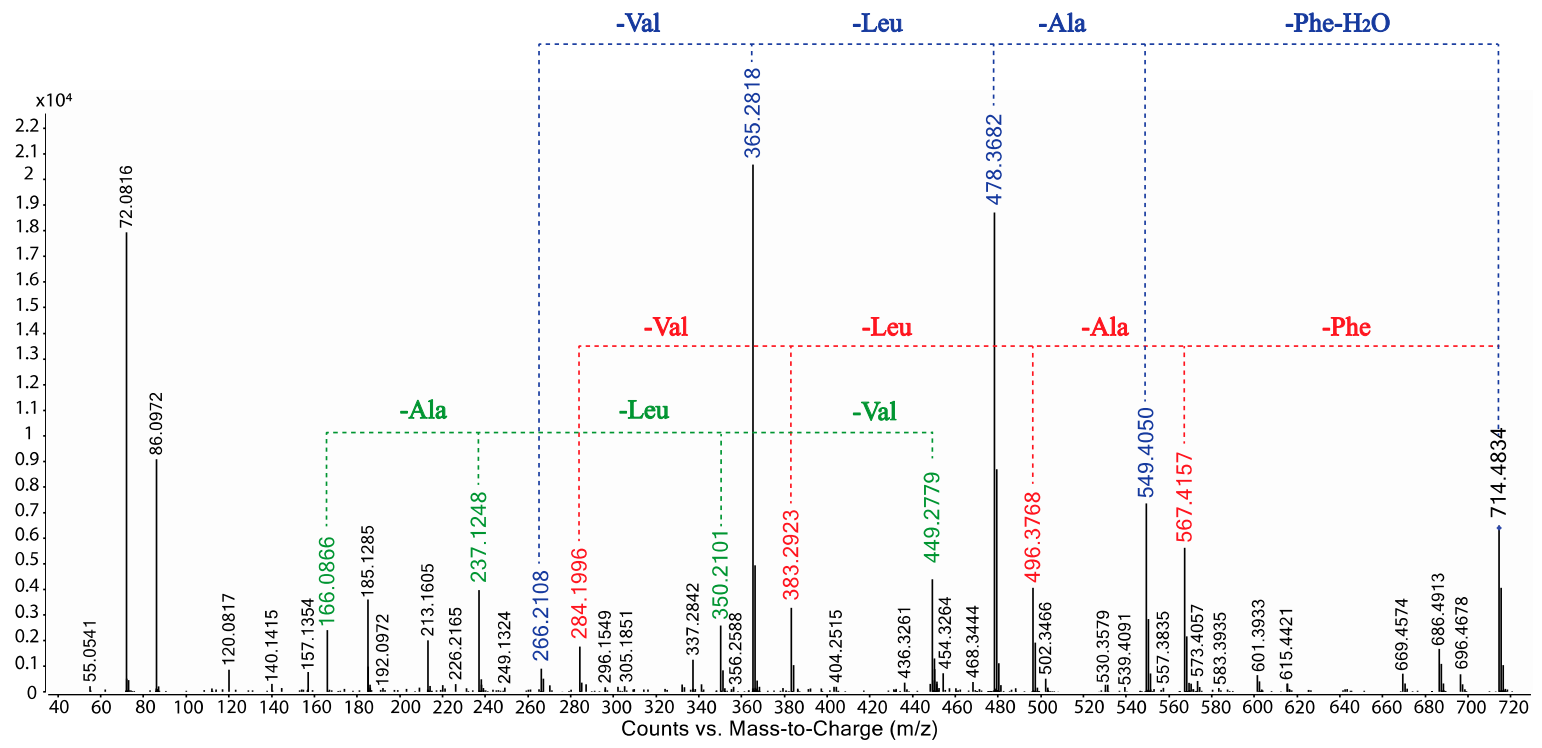

Figure 5. UPLC-QTOF-MS/MS fragmentations for scopularide C (3).
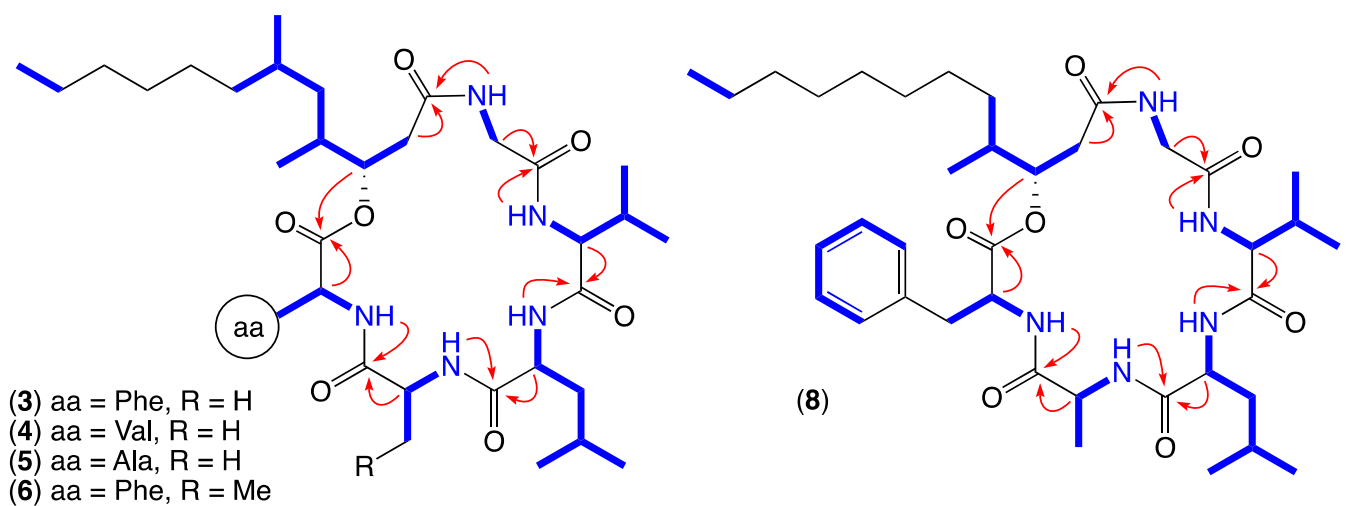

$\operatorname{COSY} \mathrm{HMBC} \curvearrowright$

Figure 6. Diagnostic 2D NMR (DMSO- $d_{6}$ ) correlations for scopularides C-F (3-6) and H (8).

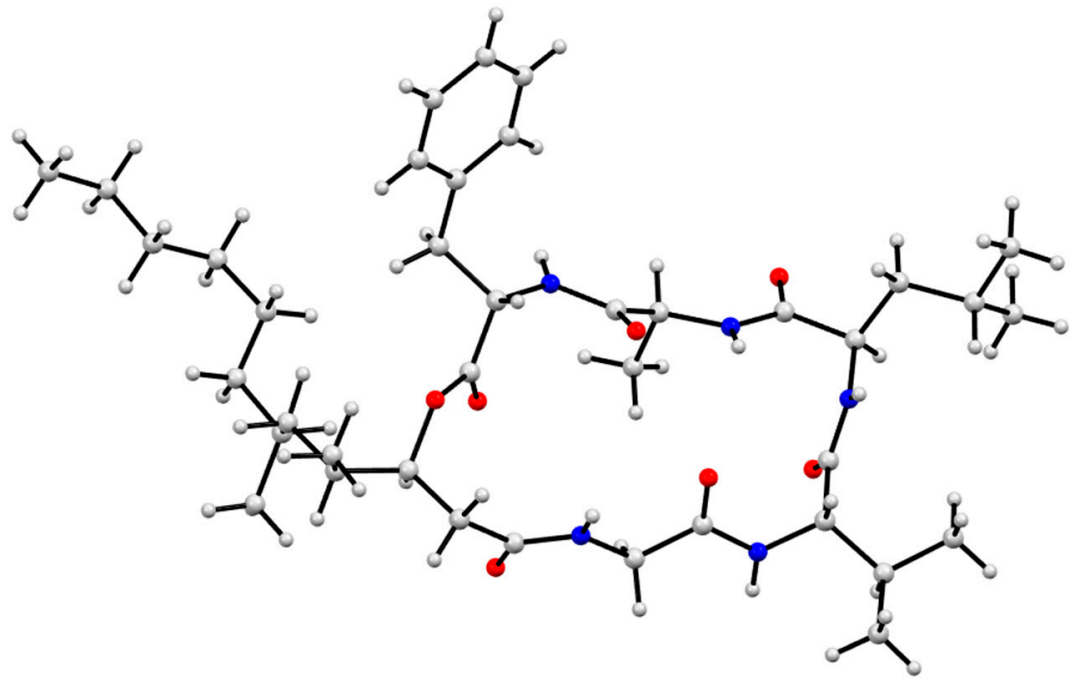

Figure 7. X-ray crystal structure of scopularide C (3). 
Table 1. 1D NMR (600 MHz, DMSO- $d_{6}$ ) data for scopularides C-E (3-5).

\begin{tabular}{|c|c|c|c|c|c|c|}
\hline \multicolumn{3}{|c|}{ (3) } & \multicolumn{2}{|l|}{ (4) } & \multicolumn{2}{|l|}{ (5) } \\
\hline Position & $\delta_{\mathrm{H}}$, mult $(J$ in $\mathrm{Hz})$ & $\delta_{C}$ & $\delta_{\mathrm{H}}$, mult $(J$ in $\mathrm{Hz})$ & $\delta_{C}$ & $\delta_{\mathrm{H}}$, mult $(J$ in $\mathrm{Hz})$ & $\delta_{C}$ \\
\hline \multicolumn{3}{|c|}{ L-Phe ${ }^{1}$} & \multicolumn{2}{|l|}{$\mathrm{L}^{-V a l^{1}}$} & \multicolumn{2}{|l|}{$\mathrm{L}^{-} \mathrm{Ala}^{1}$} \\
\hline 1 & - & 170.8 & - & 170.7 & - & $171.7^{\mathrm{g}}$ \\
\hline 2 & $4.38, \mathrm{q}(7.1)$ & 54.2 & $4.15, \mathrm{dd}(7.8,5.3)$ & 57.3 & $4.13, \mathrm{dq}(7.4,6.0)$ & 48.1 \\
\hline 3 & $\begin{array}{l}\text { a } 3.01, \mathrm{dd}(13.9,6.1) \\
\text { b } 2.93, \mathrm{~d}(13.9,8.7)\end{array}$ & 36.7 & $2.08, \mathrm{~m}$ & 29.7 & $1.28, \mathrm{~d}(7.4)$ & 16.7 \\
\hline 4 & - & 137.2 & $0.87, \mathrm{~d}(6.8)$ & 19.0 & & \\
\hline $5 / 9$ & $7.25, \mathrm{~m}$ & 129.0 & $0.85^{\mathrm{a}}, \mathrm{d}(7.0)$ & 17.4 & & \\
\hline $6 / 8$ & $7.28, \mathrm{~m}$ & 128.2 & & & & \\
\hline 7 & $7.20, \mathrm{~m}$ & 126.5 & & & & \\
\hline $\mathrm{NH}$ & $7.96, \mathrm{~d}(7.1)$ & - & 7.41, d (7.1) & - & $7.80, \mathrm{~d}(6.0)$ & - \\
\hline \multicolumn{3}{|c|}{ L-Ala ${ }^{2}$} & \multicolumn{2}{|l|}{$\mathrm{L}-\mathrm{Ala}^{2}$} & \multicolumn{2}{|l|}{$\mathrm{L}-\mathrm{Ala}^{2}$} \\
\hline 1 & - & $171.7^{\mathrm{f}}$ & - & 171.8 & - & 171.5 \\
\hline 2 & $4.16, \mathrm{dq}(7.4,7.1)$ & 47.6 & $4.19, \mathrm{dq}(8.0,7.1)$ & 47.9 & $4.18, \mathrm{dq}(7.1,6.5)$ & 47.5 \\
\hline 3 & $1.15, \mathrm{~d}(7.1)$ & 17.6 & $1.21, \mathrm{~d}(7.1)$ & 17.5 & $1.19, \mathrm{~d}(7.1)$ & 17.4 \\
\hline $\mathrm{NH}$ & $7.84, \mathrm{~d}(7.4)$ & - & $8.03, \mathrm{~d}(8.0)$ & - & $7.89, \mathrm{~d}(6.5)$ & - \\
\hline \multicolumn{3}{|c|}{ D-Leu ${ }^{3}$} & \multicolumn{2}{|l|}{ D-Leu ${ }^{3}$} & \multicolumn{2}{|l|}{ D-Leu ${ }^{3}$} \\
\hline 1 & - & 171.0 & - & 171.3 & - & 171.1 \\
\hline 2 & $4.03, \mathrm{~m}$ & 52.0 & $4.04, \mathrm{dd}(10.1,6.5)$ & 51.9 & $4.03, \mathrm{dd}(11.4,6.3)$ & 51.8 \\
\hline 3 & $1.46, \mathrm{~m}$ & 38.7 & $1.48, \mathrm{~m}$ & 38.7 & $\begin{array}{c}\text { a } 1.49, \mathrm{~m} \\
\text { b } 1.47^{\mathrm{a}}, \mathrm{m}\end{array}$ & 38.6 \\
\hline 4 & $1.63, \mathrm{~m}$ & 24.1 & $1.63, \mathrm{~m}$ & 24.1 & $1.63, \mathrm{~m}$ & 24.1 \\
\hline 5 & $0.88, \mathrm{~d}(6.5)$ & 22.9 & $0.89^{b}, d(6.7)$ & 22.9 & $0.89, \mathrm{~d}(6.7)$ & 23.0 \\
\hline 6 & $0.81, \mathrm{~d}(6.6)$ & 21.0 & $0.81, \mathrm{~d}(6.6)$ & 21.0 & $0.81, \mathrm{~d}(6.5)$ & 21.1 \\
\hline $\mathrm{NH}$ & $8.62, \mathrm{~d}(6.1)$ & - & $8.63, \mathrm{~d}(6.5)$ & - & $8.64, \mathrm{~d}(6.3)$ & - \\
\hline \multicolumn{3}{|c|}{$\mathrm{L}-\mathrm{Val}^{4}$} & \multicolumn{2}{|l|}{$\mathrm{L}^{-\mathrm{Val}^{4}}$} & \multicolumn{2}{|l|}{ L-Val ${ }^{4}$} \\
\hline 1 & - & $171.7^{\mathrm{f}}$ & - & 171.6 & - & $171.6^{\mathrm{g}}$ \\
\hline 2 & $4.10, \mathrm{dd}(8.6,7.6)$ & 58.3 & $4.06^{\mathrm{c}}, \mathrm{m}$ & 58.6 & $4.11, \mathrm{dd}(8.2,6.6)$ & 58.1 \\
\hline 3 & $1.88, \mathrm{~m}$ & 29.8 & $1.86, \mathrm{~m}$ & 29.5 & $1.85, \mathrm{~m}$ & 29.9 \\
\hline 4 & $0.87, \mathrm{~d}(6.4)$ & 18.9 & $0.88^{b}, d(6.8)$ & 19.0 & $0.87, \mathrm{~d}(6.9)$ & 18.8 \\
\hline 5 & $0.83, \mathrm{~d}(6.6)$ & 18.7 & $0.83^{\mathrm{d}}, \mathrm{d}(6.8)$ & 18.7 & $0.83, \mathrm{~d}(6.7)$ & 18.7 \\
\hline $\mathrm{NH}$ & $7.92^{a}, \mathrm{~d}(7.6)$ & - & $8.06, \mathrm{~d}(7.8)$ & - & $7.87, \mathrm{~d}(6.6)$ & - \\
\hline \multicolumn{3}{|c|}{ Gly $^{5}$} & \multicolumn{2}{|l|}{ Gly $^{5}$} & \multicolumn{2}{|l|}{ Gly $^{5}$} \\
\hline 1 & - & 168.9 & - & 169.0 & - & 168.8 \\
\hline 2 & $\begin{array}{l}\text { a } 4.07, \text { dd }(16.7,6.6) \\
\text { b } 3.41, \text { dd }(16.7,3.9)\end{array}$ & 42.3 & $\begin{array}{c}\text { a } 4.06^{\mathrm{c}}, \mathrm{m} \\
\text { b 3.44, dd }(17.1,3.8)\end{array}$ & 42.0 & $\begin{array}{l}\text { a } 4.06, \mathrm{dd}(16.5,6.6) \\
\text { b } 3.43, \mathrm{dd}(16.5,4.0)\end{array}$ & 42.3 \\
\hline $\mathrm{NH}$ & $7.91^{\mathrm{a}}, \mathrm{dd}(6.5,3.9)$ & - & $7.90, \mathrm{dd}(5.8,3.8)$ & - & $7.82, \mathrm{dd}(6.1,4.0)$ & - \\
\hline \multicolumn{3}{|c|}{ HDMLA } & \multicolumn{2}{|l|}{ HDMLA } & \multicolumn{2}{|l|}{ HDMLA } \\
\hline $1^{\prime}$ & - & 169.8 & - & 170.0 & - & 169.8 \\
\hline $2^{\prime}$ & $\begin{array}{l}\text { a 2.51, dd }(15.2,9.8) \\
\text { b 2.25, d }(15.2,1.4)\end{array}$ & 37.7 & $\begin{array}{l}\text { a } 2.52, \mathrm{dd}(14.5,9.6) \\
\text { b } 2.23, \mathrm{dd}(14.5,1.4)\end{array}$ & 38.0 & $\begin{array}{l}\text { a } 2.53, \mathrm{dd}(14.7,10.1) \\
\text { b } 2.24, \mathrm{dd}(14.7,1.8)\end{array}$ & 37.7 \\
\hline $3^{\prime}$ & 4.94 , ddd $(9.1,5.1,1.8)$ & 74.8 & 4.92 , ddd $(9.3,4.8,1.4)$ & 75.2 & 4.97, ddd $(10.1,4.8,1.8)$ & 74.2 \\
\hline $4^{\prime}$ & $1.68, \mathrm{~m}$ & 33.7 & $1.79, \mathrm{~m}$ & 33.9 & $1.75, \mathrm{~m}$ & 33.8 \\
\hline $5^{\prime}$ & $\begin{array}{l}\text { a } 1.18^{\mathrm{b}}, \mathrm{m} \\
\mathrm{b} 0.75, \mathrm{~m}\end{array}$ & $39.5^{\mathrm{h}}$ & $\begin{array}{l}\text { a } 1.29, \mathrm{~m} \\
\text { b } 0.87, \mathrm{~m}\end{array}$ & $39.6^{\mathrm{h}}$ & $\begin{array}{l}\text { a } 1.26, \mathrm{~m} \\
\text { b } 0.84, \mathrm{~m}\end{array}$ & $39.6^{\mathrm{h}}$ \\
\hline $6^{\prime}$ & $1.41, \mathrm{~m}$ & 29.3 & $1.44, \mathrm{~m}$ & 29.3 & $1.46^{\mathrm{a}}, \mathrm{m}$ & 29.2 \\
\hline $7^{\prime}$ & $\begin{array}{l}\text { a } 1.19^{\mathrm{b}}, \mathrm{m} \\
\text { b } 0.95, \mathrm{~m}\end{array}$ & 35.7 & $\begin{array}{l}\text { a } 1.22 \mathrm{e}, \mathrm{m} \\
\text { b } 0.98, \mathrm{~m}\end{array}$ & 36.0 & $\begin{array}{l}\text { a } 1.23^{\mathrm{b}}, \mathrm{m} \\
\text { b } 0.99, \mathrm{~m}\end{array}$ & 35.8 \\
\hline $8^{\prime}$ & $1.20^{\mathrm{b}}, \mathrm{m}$ & 26.1 & $\begin{array}{l}\text { a } 1.26, \mathrm{~m} \\
\text { b } 1.16, \mathrm{~m}\end{array}$ & 26.1 & $\begin{array}{l}\text { a } 1.24, \mathrm{~m} \\
\text { b } 1.16, \mathrm{~m}\end{array}$ & 26.1 \\
\hline $9^{\prime}$ & $1.22^{\mathrm{c}}, \mathrm{m}$ & 29.1 & $\begin{array}{l}\text { a } 1.22^{\mathrm{e}}, \mathrm{m} \\
\mathrm{b} 1.19, \mathrm{~m}\end{array}$ & 29.1 & $1.23^{\mathrm{b}}, \mathrm{m}$ & 29.0 \\
\hline $10^{\prime}$ & $1.22^{\mathrm{c}}, \mathrm{m}$ & 31.3 & $1.23^{\mathrm{e}}, \mathrm{m}$ & 31.3 & $1.23^{\mathrm{b}}, \mathrm{m}$ & 31.3 \\
\hline $11^{\prime}$ & $1.24, \mathrm{~m}$ & 22.1 & $1.25, \mathrm{~m}$ & 22.1 & $1.26, \mathrm{~m}$ & 22.1 \\
\hline $12^{\prime}$ & $0.84, \mathrm{t}(6.9)$ & 13.9 & $0.85^{\mathrm{a}}, \mathrm{t}(7.9)$ & 13.9 & $0.85, \mathrm{t}(7.1)$ & 13.9 \\
\hline $4^{\prime}-\mathrm{Me}$ & $0.70, \mathrm{~d}(6.7)$ & 14.8 & $0.84^{\mathrm{d}}, \mathrm{d}(8.0)$ & 15.1 & $0.82, \mathrm{~d}(6.4)$ & 15.0 \\
\hline $6^{\prime}-\mathrm{Me}$ & $0.80, \mathrm{~d}(6.6)$ & 20.2 & $0.83^{\mathrm{d}}, \mathrm{d}(7.4)$ & 20.0 & $0.83, \mathrm{~d}(6.7)$ & 20.2 \\
\hline
\end{tabular}

${ }^{\mathrm{a}-\mathrm{e}}$ Overlapping resonances within the same letter and column, ${ }^{\mathrm{f}-\mathrm{g}}$ assignments are interchangeable within the same letter and column, ${ }^{\mathrm{h}}$ obscured by solvent. HDMLA $=3 S, 4 S, 6 S$-3-hydroxyl-4,6-dimethyllauric acid 
HRESI(+)MS analysis of scopularide D (4) revealed a molecular formula $\left(\mathrm{C}_{35} \mathrm{H}_{63} \mathrm{~N}_{5} \mathrm{O}_{7}\right)$ consistent with an L-Phe to L-Val substitution analogue of 3, with $\mathrm{C}_{3}$ Marfey's analysis (Figure S49) supported by diagnostic of MS/MS fragmentations (Figure S40) and 1D and 2D NMR (DMSO- $d_{6}$ ) data (Table 1 and Table S6, Figure 6 and Figures S19-S23) establishing the amino acid sequence L-Val ${ }^{1}-\mathrm{L}-\mathrm{Ala}^{2}-\mathrm{D}-\mathrm{Leu}^{3}-\mathrm{L}-\mathrm{Val}^{4}-\mathrm{Gly}^{5}$. Similarly, HRESI(+)MS analysis of scopularide E (5) revealed a molecular formula $\left(\mathrm{C}_{33} \mathrm{H}_{59} \mathrm{~N}_{5} \mathrm{O}_{7}\right)$ consistent with an L-Phe to L-Ala substitution analogue of 3 , with $\mathrm{C}_{3}$ Marfey's analysis (Figure S50) supported by diagnostic of MS/MS fragmentations (Figure S41) and 1D and 2D NMR (DMSO- $d_{6}$ ) data (Table 1 and Table S7, Figure 6 and Figures S24-S28) establishing the amino acid sequence $\mathrm{L}-\mathrm{Ala}^{1}-\mathrm{L}-\mathrm{Ala}^{2}-\mathrm{D}-\mathrm{Leu}^{3}-\mathrm{L}-\mathrm{Val}^{4}-\mathrm{Gly}^{5}$. Likewise, HRESI(+)MS analysis of scopularide $\mathrm{F}$ (6) revealed a molecular formula $\left(\mathrm{C}_{40} \mathrm{H}_{65} \mathrm{~N}_{5} \mathrm{O}_{7}\right)$ consistent with a homologue $\left(+\mathrm{CH}_{2}\right)$ of 3 , with $\mathrm{C}_{3}$ Marfey's analysis (Figure S51) supported by diagnostic of MS/MS fragmentations (Figure S42) and 1D and 2D NMR (DMSO- $d_{6}$ ) data (Table 2 and Table S8, Figure 6 and Figures S29-S33) establishing the amino acid sequence L-Phe ${ }^{1}-\mathrm{L}-\mathrm{Abu}^{2}-\mathrm{D}-\mathrm{Leu}^{3}-\mathrm{L}-\mathrm{Val}^{4}-\mathrm{Gly}^{5}$ (where $\mathrm{L}$-Abu is $\mathrm{L}$-alpha-aminobutyric acid). Importantly, the structure and absolute configuration of the lipid residue in 4-6 were assigned on the basis of spectroscopic comparisons with $\mathbf{3}$, and biosynthetic considerations.

Although scopularide G (7) was only isolated at yields that precluded the acquisition of 1D and 2D NMR data, HRESI(+)MS analysis revealed a molecular formula $\left(\mathrm{C}_{36} \mathrm{H}_{65} \mathrm{~N}_{5} \mathrm{O}_{7}\right)$ consistent with an L-Phe to L-Val substitution analogue of 6 , with $C_{3}$ Marfey's analysis (Figure S52) supported by diagnostic of MS/MS fragmentations (Figures S43 and S44) establishing the amino acid sequence $\mathrm{L}-\mathrm{Val}^{1}-\mathrm{L}-\mathrm{Abu}^{2}-\mathrm{D}-\mathrm{Leu}^{3}-\mathrm{L}-\mathrm{Val}^{4}-\mathrm{Gly}^{5}$. Based on biosynthetic considerations, the structure and absolute configuration of the lipid residue in 7 was proposed to be in common with 3 . Although the putative scopularide H (8) could be detected in the GNPS analysis of Beauveria sp. CMB-F585 (Figures 2 and 3), its isolation and structure elucidation were more readily achieved from Scopulariopsis sp. CMB-F115 (Figure 2).

GNPS analysis of the EtOAc extract obtained from a YES solid phase $(40 \times$ agar plate) cultivation of Scopulariopsis sp. CMB-F115 detected the presence of the prominent new scopularide $\mathrm{H}(8)$ and the known scopularide A (1) (Figure 8). Solvent extraction and partitioning of the EtOAc extract, followed by reversed-phase HPLC, yielded the known scopularide A (1) together with the new analogue 8. HRESI(+)MS analysis of scopularide $\mathrm{H}(8)$ revealed a molecular formula $\left(\mathrm{C}_{38} \mathrm{H}_{61} \mathrm{~N}_{5} \mathrm{O}_{7}\right)$ consistent with a lower homologue $\left(-\mathrm{CH}_{2}\right)$ of 3 . A diagnostic of MS/MS fragmentations (Figure S45) and 1D and 2D NMR (DMSO- $d_{6}$ ) (Table 2 and Table S9, Figure 6 and Figures S34-38) established the amino acid sequence $\mathrm{Phe}^{1}-\mathrm{Ala}^{2}-\mathrm{Leu}^{3}-\mathrm{Val}^{4}-\mathrm{Gly}^{5}$. Finally, the complete structure for scopularide H (8), including absolute configuration about the amino acids and 3S,4S-3-hydroxyl-4-methyllauric acid (3S,4S- HMLA) lipid residue, was confirmed by single crystal X-ray analysis (Figure 9 and Figure S56).

Table 2. 1D NMR (600 MHz, DMSO- $d_{6}$ ) data for scopularides $\mathrm{F}$ and $\mathrm{H}$ (6 and 8).

\begin{tabular}{|c|c|c|c|c|}
\hline \multirow[b]{2}{*}{ Position } & \multicolumn{2}{|l|}{ (6) } & \multicolumn{2}{|l|}{ (8) } \\
\hline & $\delta_{\mathrm{H}}$, mult $(J$ in $\mathrm{Hz})$ & $\delta_{C}$ & $\delta_{\mathrm{H}}$, mult $(J$ in $\mathrm{Hz})$ & $\delta_{C}$ \\
\hline & \multicolumn{2}{|l|}{ L-Phe ${ }^{1}$} & \multicolumn{2}{|l|}{ L-Phe ${ }^{1}$} \\
\hline 1 & - & 170.9 & - & 170.8 \\
\hline 2 & $4.38, \mathrm{~m}$ & 54.3 & $4.34, \mathrm{q}(7.4)$ & 54.5 \\
\hline 3 & $\begin{array}{l}\text { a } 2.97, \text { dd }(13.9,6.1) \\
\text { b } 2.95, \text { dd }(13.9,8.4)\end{array}$ & 36.7 & $2.98, \mathrm{~m}$ & 36.7 \\
\hline 4 & - & 137.1 & - & 137.2 \\
\hline $5 / 9$ & $7.26, \mathrm{~m}$ & 128.9 & $7.26, \mathrm{~m}$ & 129.1 \\
\hline $6 / 8$ & $7.28, \mathrm{~m}$ & 128.3 & $7.26, \mathrm{~m}$ & 128.2 \\
\hline 7 & $7.21, \mathrm{~m}$ & 126.6 & $7.20, \mathrm{~m}$ & 126.5 \\
\hline $\mathrm{NH}$ & $8.14, \mathrm{~d}(6.5)$ & - & $8.03, \mathrm{~d}(6.7)$ & - \\
\hline
\end{tabular}


Table 2. Cont.

\begin{tabular}{|c|c|c|c|c|}
\hline \multirow[b]{2}{*}{ Position } & \multicolumn{2}{|l|}{ (6) } & \multicolumn{2}{|l|}{ (8) } \\
\hline & $\delta_{\mathrm{H}}$, mult $(J$ in $\mathrm{Hz})$ & $\delta_{C}$ & $\delta_{\mathrm{H}}$, mult $(J$ in $\mathrm{Hz})$ & $\delta_{C}$ \\
\hline & \multicolumn{2}{|l|}{$\mathrm{L}-\mathrm{Abu}^{2}$} & \multicolumn{2}{|l|}{ L-Ala ${ }^{2}$} \\
\hline 1 & - & 171.0 & - & 171.8 \\
\hline 2 & $4.08^{\mathrm{a}}, \mathrm{m}$ & 52.9 & $4.19, \mathrm{dq}(8.1,7.1)$ & 47.6 \\
\hline 3 & $\begin{array}{l}\text { a } 1.74, \mathrm{~m} \\
\text { b } 1.48^{\mathrm{b}}, \mathrm{m}\end{array}$ & 24.4 & $1.15, \mathrm{~d}(7.1)$ & 17.6 \\
\hline 4 & $0.73, \mathrm{t}(7.2)$ & 9.5 & & \\
\hline \multirow[t]{2}{*}{$\mathrm{NH}$} & $7.67, \mathrm{~d}(8.0)$ & - & $7.83, \mathrm{~d}(8.1)$ & - \\
\hline & \multicolumn{2}{|l|}{ D-Leu ${ }^{3}$} & D-Leu ${ }^{3}$ & \\
\hline 1 & - & 171.1 & - & 171.0 \\
\hline 2 & $4.09^{\mathrm{a}}, \mathrm{m}$ & 51.8 & $4.03, \mathrm{~m}$ & 52.0 \\
\hline 3 & $1.47^{\mathrm{b}}, \mathrm{m}$ & 38.6 & $\begin{array}{l}\text { a } 1.47, \mathrm{~m} \\
\text { b } 1.44, \mathrm{~m}\end{array}$ & 38.7 \\
\hline 4 & $1.61, \mathrm{~m}$ & 24.1 & $1.63, \mathrm{~m}$ & 24.1 \\
\hline 5 & $0.88, \mathrm{~d}(6.5)$ & 22.8 & $0.89, \mathrm{~d}(6.5)$ & 22.9 \\
\hline 6 & $0.81, \mathrm{~d}(6.8)$ & 21.2 & $0.82^{\mathrm{d}}, \mathrm{d}(6.5)$ & 21.1 \\
\hline \multirow[t]{2}{*}{$\mathrm{NH}$} & $8.62, \mathrm{~d}(6.3)$ & - & $8.63, \mathrm{~d}(6.0)$ & - \\
\hline & \multicolumn{2}{|l|}{$\mathrm{L}^{-\mathrm{Val}^{4}}$} & $\mathrm{~L}^{-\mathrm{Val}^{4}}$ & \\
\hline 1 & - & 171.6 & - & 171.7 \\
\hline 2 & $4.15, \mathrm{dd}(8.9,8.7)$ & 58.1 & $4.10, \mathrm{dd}(8.6,7.9)$ & 58.3 \\
\hline 3 & $1.88, \mathrm{~m}$ & 29.9 & $1.87, \mathrm{~m}$ & 29.8 \\
\hline 4 & $0.86, \mathrm{~d}(6.7)$ & 18.8 & $0.88^{c}, \mathrm{~d}(6.2)$ & 18.9 \\
\hline 5 & $0.83^{f}, d(6.6)$ & 18.7 & $0.81^{\mathrm{d}}, \mathrm{d}(6.1)$ & 18.7 \\
\hline \multirow[t]{2}{*}{$\mathrm{NH}$} & $7.86, \mathrm{~d}(8.9)$ & - & $7.88, \mathrm{~d}(7.9)$ & - \\
\hline & \multicolumn{2}{|l|}{ Gly $^{5}$} & \multicolumn{2}{|l|}{ Gly ${ }^{5}$} \\
\hline 1 & - & 168.6 & - & 168.9 \\
\hline 2 & $\begin{array}{l}\text { a } 4.01, \mathrm{dd}(16.7,6.3) \\
\text { b } 3.42, \mathrm{dd}(16.7,4.7)\end{array}$ & 42.4 & $\begin{array}{l}\text { a } 4.07, \mathrm{dd}(16.6,6.4) \\
\text { b } 3.40, \mathrm{dd}(16.6,4.1)\end{array}$ & 42.3 \\
\hline \multirow[t]{2}{*}{$\mathrm{NH}$} & 7.85, dd $(5.6,4.7)$ & - & 7.93, dd $(6.4,4.1)$ & - \\
\hline & \multicolumn{2}{|l|}{ HDMLA } & HMLA & \\
\hline $1^{\prime}$ & - & 169.8 & - & 169.8 \\
\hline $2^{\prime}$ & $\begin{array}{l}\text { a } 2.53, \text { dd }(14.8,9.4) \\
\text { b } 2.24, \text { dd }(14.8,1.2)\end{array}$ & 37.9 & $\begin{array}{l}\text { a } 2.48, \text { dd }(14.9,9.2) \\
\text { b } 2.24, \text { dd }(14.9,1.8)\end{array}$ & 37.0 \\
\hline $3^{\prime}$ & $4.94, \operatorname{ddd}(9.1,3.2,1.8)$ & 74.7 & 4.90, ddd $(9.2,5.7,1.8)$ & 75.1 \\
\hline $4^{\prime}$ & $1.66, \mathrm{~m}$ & 33.9 & $1.50, \mathrm{~m}$ & 36.1 \\
\hline $5^{\prime}$ & $\begin{array}{l}\text { a } 1.17^{\mathrm{e}}, \mathrm{m} \\
\text { b } 0.75, \mathrm{~m}\end{array}$ & $39.6^{\mathrm{f}}$ & $\begin{array}{l}\text { a } 1.19^{b}, m \\
\text { b } 0.87^{c}, m\end{array}$ & 31.4 \\
\hline $6^{\prime}$ & $1.41, \mathrm{~m}$ & 29.2 & $\begin{array}{l}\text { a } 1.19^{b}, \mathrm{~m} \\
\text { b } 1.09, \mathrm{~m}\end{array}$ & 26.5 \\
\hline $7^{\prime}$ & $\begin{array}{l}\text { a } 1.19, \mathrm{~m} \\
\text { b } 0.95, \mathrm{~m}\end{array}$ & 35.8 & $1.18^{\mathrm{b}}, \mathrm{m}$ & 29.3 \\
\hline $8^{\prime}$ & $\begin{array}{l}\text { a } 1.25^{\mathrm{c}}, \mathrm{m} \\
\mathrm{b} 1.17^{\mathrm{e}}, \mathrm{m}\end{array}$ & 26.1 & $1.23^{\mathrm{a}}, \mathrm{m}$ & 28.9 \\
\hline $9^{\prime}$ & $1.22^{\mathrm{d}}, \mathrm{m}$ & 29.1 & $1.23^{\mathrm{a}}, \mathrm{m}$ & 28.7 \\
\hline $10^{\prime}$ & $1.22^{\mathrm{d}}, \mathrm{m}$ & 31.3 & $1.23^{\mathrm{a}}, \mathrm{m}$ & 31.3 \\
\hline $11^{\prime}$ & $1.24^{\mathrm{c}}, \mathrm{m}$ & 22.1 & $1.25, \mathrm{~m}$ & 22.1 \\
\hline $12^{\prime}$ & $0.83^{f}, t(6.9)$ & 13.9 & $0.85, \mathrm{t}(6.6)$ & 13.9 \\
\hline $4^{\prime}-\mathrm{Me}$ & $0.69, \mathrm{~d}(6.7)$ & 14.8 & $0.67, \mathrm{~d}(6.9)$ & 14.5 \\
\hline $6^{\prime}-\mathrm{Me}$ & $0.80, \mathrm{~d}(6.8)$ & 20.1 & & \\
\hline
\end{tabular}

a-e Overlapping resonances within the same letter and column, $\mathrm{f}$ obscured by solvent. HDMLA = 3S,4S,6S-3-hydroxyl-4,6-dimethyllauric acid. HMLA = 3S,4S-3-hydroxyl-4-methyllauric acid. 


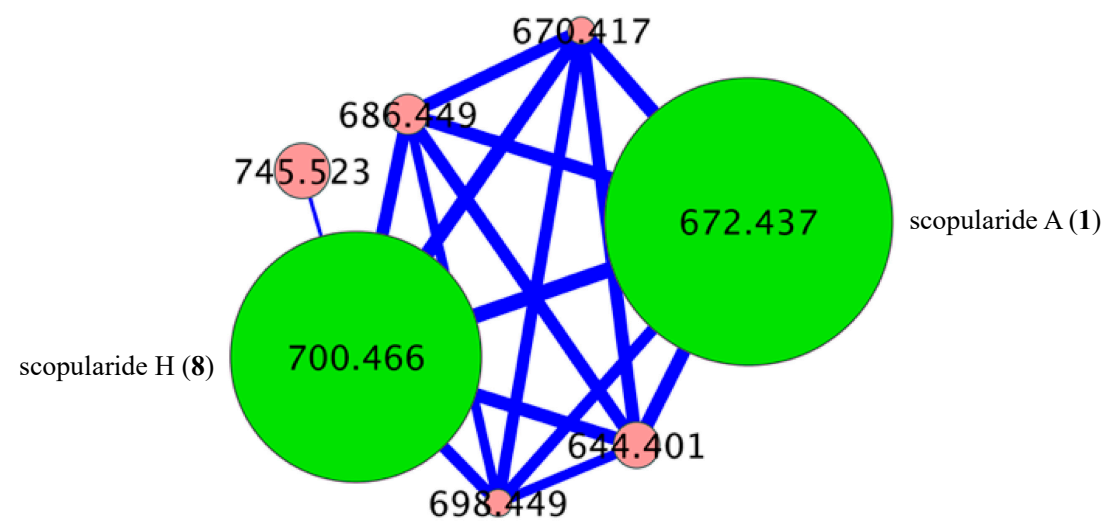

Figure 8. GNPS cluster for scopularides detected in a YES solid phase cultivation of Scopulariopsis sp. CMB-F115. Green nodes are scopularides A (1) and H (8), and pink nodes are minor analogues.

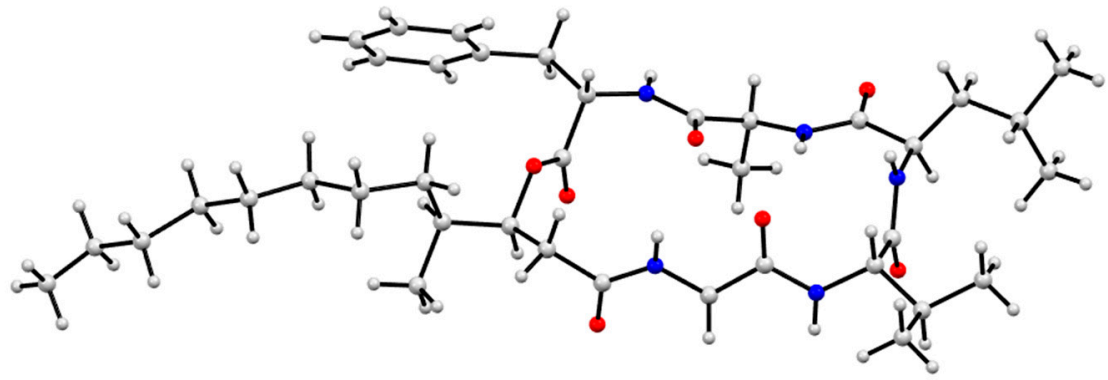

Figure 9. X-ray crystal structure of scopularide H (8).

The scopularides A-H (1-8) did not exhibit any significant growth inhibition $\left(\mathrm{IC}_{50}>30 \mu \mathrm{M}\right)$ when tested against drug sensitive Gram -ve Escherichia coli ATCC 11775 and Gram +ve Staphylococcus aureus ATCC 9144, or clinical isolates of both methicillin-susceptible and methicillin-resistant Staphylococcus aureus, and a clinical isolate of vancomycin-resistant Enterococcus faecalis. Likewise, 1-8 were not cytotoxic towards the fungus Candida albicans ATCC 10231 or human colorectal (SW620), lung (NCI-H460) or hepatocellular (HepG2) carcinoma cells.

Literature accounts of scopularides and related lipodepsipeptides are limited to scopularides A-B (1-2) from the marine sponge-derived fungus Scopulariopsis brevicaulis NCPF-2177 [2]; oryzamides A-E from the marine sponge-derived fungus Nigrospora oryzae PF18 [5]; chrysogeamides A-G from the marine coral-derived Penicillium chrysogenum CHNSCLM-0003 [6]; emericellamides A-B from the marine green alga-derived fungus Emericella sp. CNL-878 co-cultured with the marine sediment-derived actinomycete Salinispora arenicola CNH-665 [7]; emericellamides C-F from the non-marine Aspergillus nidulans [8]; nodupetide from the insect-derived fungus Nodulisporum sp. IFB-A163 [9]; as well as numerous isariins and iso-isariins from Isaria spp. and Beauveria felina [10-17]. Somewhat surprisingly, they also include arenamides A-C from the marine sediment-derived actinomycete Salinispora arenicola CNT-088 [18]. A summary of the amino acid and lipid residue compositions of these metabolites is presented in Tables 3 and 4.

Notwithstanding the high level of homology shown across Tables 3 and 4, in addition to comparing the 1D NMR (methanol- $d_{4}$ ) data for our re-isolated samples of $\mathbf{1}$ and $\mathbf{2}$ with literature data (Tables S1 and S3) [2], we were surprised to find an equally good match of the 1D NMR (DMSO- $d_{6}$ ) data for 1 and 2 with that reported for arenamides A-B (Tables S2-S4) [18,20]. Whether this latter observation is coincidence, or indicative of a common D-Leu ${ }^{3}$ configuration, remains unclear. 
Table 3. Amino acid sequence homology for natural lipodepsipeptides related to scopularides.

\begin{tabular}{|c|c|c|c|c|c|c|c|c|c|c|c|}
\hline Compound & $\mathbf{a a}^{1}$ & $\mathrm{aa}^{2}$ & $\mathrm{aa}^{3}$ & $\mathbf{a a}^{4}$ & $\mathrm{aa}^{5}$ & Compound & $\mathrm{aa}^{1}$ & $\mathrm{aa}^{2}$ & $\mathrm{aa}^{3}$ & $\mathbf{a a}^{4}$ & $a^{5}{ }^{5}$ \\
\hline scopularide A [2] & L-Phe & L-Ala & D-Leu & L-Val & Gly & iso-isariin D [17] & L-Ala & L-Ala & D-Leu & L-Val & Gly \\
\hline scopularide B [2] & L-Phe & L-Ala & D-Leu & L-Val & Gly & isariin G2 [15] & L-Ala & L-Ala & D-Leu & L-Val & Gly \\
\hline scopularide C & L-Phe & L-Ala & D-Leu & L-Val & Gly & scopularide E & L-Ala & L-Ala & D-Leu & L-Val & Gly \\
\hline scopularide $\mathrm{H}$ & L-Phe & L-Ala & D-Leu & L-Val & Gly & isariin F1 [15] & $\mathrm{Abu} / \mathrm{Aib}$ & L-Ala & D-Leu & L-Val & Gly \\
\hline chrysogeamide A [6] & L-Val & L-Ala & D-Leu & L-Val & Gly & scopularide F & L-Phe & $\mathrm{L}-\mathrm{Abu}$ & D-Leu & L-Val & Gly \\
\hline chrysogeamide B [6] & L-Val & L-Ala & D-Leu & L-Val & Gly & chrysogeamide C [6] & L-Phe & L-Ala & D-Leu & D-Leu & Gly \\
\hline scopularide D & L-Val & L-Ala & D-Leu & L-Val & Gly & chrysogeamide D [6] & L-Phe & L-Ala & D-Leu & D-Leu & Gly \\
\hline nodupetide [9] & L-Val & L-Ala & D-Leu & L-Val & Gly & chrysogeamide E [6] & L-Phe & L-Ala & D-Leu & D-Leu & Gly \\
\hline isariin $\mathrm{A}[10,11]$ & L-Val & L-Ala & D-Leu & L-Val & Gly & chrysogeamide F [6] & L-Phe & L-Ala & D-Leu & L-Pro & Gly \\
\hline isariin B [12-14] & L-Val & L-Ala & D-Leu & L-Val & Gly & chrysogeamide G [6] & L-Phe & L-Ala & D-Leu & L-Pro & Gly \\
\hline iso-isariin B [16] & L-Val & L-Ala & D-Leu & L-Val & Gly & scopularide G & L-Val & $\mathrm{L}-\mathrm{Abu}$ & D-Leu & L-Val & Gly \\
\hline isariin C2 [15] & L-Val & L-Ala & D-Leu & L-Val & Gly & emericellamide A [7] & L-Ala & L-Ala & L-Leu & L-Val & Gly \\
\hline isariin E [15] & L-Val & L-Ala & D-Leu & L-Val & Gly & emericellamide B [7] & L-Ala & L-Ala & L-Leu & L-Val & Gly \\
\hline isariin F2 [15] & L-Val & L-Ala & D-Leu & L-Val & Gly & emericellamide C [8] & L-Ala & L-Ala & L-Leu & L-Val & Gly \\
\hline isariin G1 [15] & L-Val & L-Ala & D-Leu & L-Val & Gly & emericellamide D [8] & L-Ala & L-Ala & L-Leu & L-Val & Gly \\
\hline oryzamide A [5] & L-Leu & L-Ala & D-Leu & L-Val & Gly & emericellamide E [8] & L-Ala & L-Ala & L-Leu & L-Val & Gly \\
\hline oryzamide B [5] & L-Tyr & L-Ala & D-Leu & L-Val & Gly & emericellamide F [8] & L-Ala & L-Ala & L-Leu & L-Val & Gly \\
\hline oryzamide C [5] & L-Met & L-Ala & D-Leu & L-Val & Gly & arenamide A [18] & L-Phe & L-Ala & L-Leu & L-Val & Gly \\
\hline isariin C $[13,14]$ & L-Ala & L-Ala & D-Leu & L-Val & Gly & arenamide B [18] & L-Phe & L-Ala & L-Leu & L-Val & Gly \\
\hline isariin D $[13,14]$ & L-Ala & L-Ala & D-Leu & L-Val & Gly & arenamide C [18] & L-Met & L-Ala & L-Leu & L-Val & Gly \\
\hline
\end{tabular}


Table 4. Lipid residue homology for natural lipodepsipeptides related to scopularides.

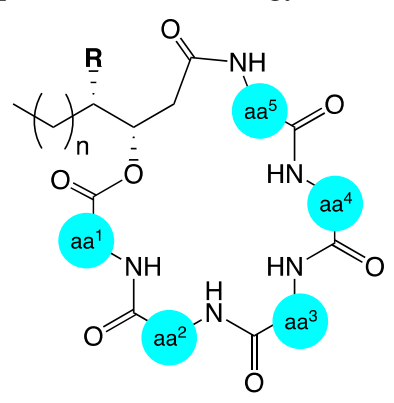

(a)

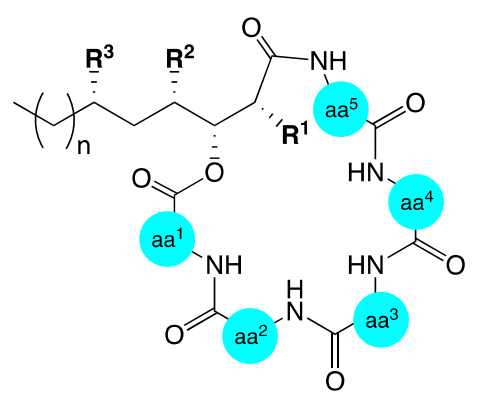

(b)

\begin{tabular}{|c|c|c|c|c|c|c|c|c|c|}
\hline \multicolumn{10}{|c|}{ Structure a } \\
\hline compound & $\mathbf{n}$ & $\mathbf{R}$ & & & compound & $\mathbf{n}$ & $\mathbf{R}$ & & \\
\hline isariin $E^{*}[15]$ & 1 & $\mathrm{H}$ & & & iso-isariin $B$ * [16] & 3 & $\mathrm{Me}$ & & \\
\hline iso-isariin D [17] & 1 & $\mathrm{Me}$ & & & isariin $B$ [12-14] & 4 & $\mathrm{H}$ & & \\
\hline nodupetide [9] & 1 & $\mathrm{Me}$ & & & isariin $C^{*}[13,14]$ & 4 & $\mathrm{H}$ & & \\
\hline chrysogeamide D [6] & 1 & $\mathrm{Me}$ & & & isariin $\mathrm{F} 1 *[15]$ & 4 & $\mathrm{H}$ & & \\
\hline isariin $C 2 *[15]$ & 2 & $\mathrm{H}$ & & & isariin $\mathrm{G} 1 *[15]$ & 5 & $\mathrm{H}$ & & \\
\hline isariin $\mathrm{D} *[13,14]$ & 2 & $\mathrm{H}$ & & & isariin $\mathrm{A}[10,11,19]$ & 7 & $\mathrm{H}$ & & \\
\hline isariin F2 * [15] & 3 & $\mathrm{H}$ & & & isariin $\mathrm{G} 2 *[15]$ & 7 & $\mathrm{H}$ & & \\
\hline \multicolumn{10}{|c|}{ Structure b } \\
\hline compound & $\mathbf{n}$ & $\mathbf{R}^{1}$ & $\mathbf{R}^{2}$ & $\mathrm{R}^{3}$ & compound & $\mathbf{n}$ & $\mathbf{R}^{1}$ & $\mathbf{R}^{2}$ & $\mathbf{R}^{3}$ \\
\hline scopularide B [2] & 1 & $\mathrm{H}$ & $\mathrm{Me}$ & $\mathrm{H}$ & arenamide A $[18,20]$ & 3 & $\mathrm{H}$ & $\mathrm{Me}$ & $\mathrm{H}$ \\
\hline chrysogeamide A [6] & 1 & $\mathrm{H}$ & $\mathrm{Me}$ & $\mathrm{H}$ & arenamide $C[18,20]$ & 3 & $\mathrm{H}$ & $\mathrm{Me}$ & $\mathrm{H}$ \\
\hline chrysogeamide E [6] & 1 & $\mathrm{H}$ & $\mathrm{Me}$ & $\mathrm{H}$ & emericellamide A [7] & 3 & $\mathrm{Me}$ & $\mathrm{Me}$ & $\mathrm{H}$ \\
\hline chrysogeamide F [6] & 1 & $\mathrm{H}$ & $\mathrm{Me}$ & $\mathrm{H}$ & emericellamide C [8] & 3 & $\mathrm{Me}$ & $\mathrm{H}$ & $\mathrm{H}$ \\
\hline arenamide B $[18,20]$ & 1 & $\mathrm{H}$ & $\mathrm{Me}$ & $\mathrm{H}$ & emericellamide E [8] & 5 & $\mathrm{Me}$ & $\mathrm{H}$ & $\mathrm{H}$ \\
\hline scopularide A [2] & 3 & $\mathrm{H}$ & $\mathrm{Me}$ & $\mathrm{H}$ & emericellamide F [8] & 5 & $\mathrm{H}$ & $\mathrm{Me}$ & $\mathrm{H}$ \\
\hline chrysogeamide B [6] & 3 & $\mathrm{H}$ & $\mathrm{Me}$ & $\mathrm{H}$ & scopularide $\mathrm{H}$ & 5 & $\mathrm{H}$ & $\mathrm{Me}$ & $\mathrm{H}$ \\
\hline chrysogeamide $C[6]$ & 3 & $\mathrm{H}$ & $\mathrm{Me}$ & $\mathrm{H}$ & scopularide $\mathrm{C}$ & 5 & $\mathrm{H}$ & $\mathrm{Me}$ & $\mathrm{Me}$ \\
\hline chrysogeamide G [6] & 3 & $\mathrm{H}$ & $\mathrm{Me}$ & $\mathrm{H}$ & scopularide D & 5 & $\mathrm{H}$ & $\mathrm{Me}$ & $\mathrm{Me}$ \\
\hline oryzamide A [5] & 3 & $\mathrm{H}$ & $\mathrm{Me}$ & $\mathrm{H}$ & scopularide E & 5 & $\mathrm{H}$ & $\mathrm{Me}$ & $\mathrm{Me}$ \\
\hline oryzamide B [5] & 3 & $\mathrm{H}$ & $\mathrm{Me}$ & $\mathrm{H}$ & scopularide F & 5 & $\mathrm{H}$ & $\mathrm{Me}$ & $\mathrm{Me}$ \\
\hline oryzamide C [5] & 3 & $\mathrm{H}$ & $\mathrm{Me}$ & $\mathrm{H}$ & scopularide $\mathrm{G}$ & 5 & $\mathrm{H}$ & $\mathrm{Me}$ & $\mathrm{Me}$ \\
\hline emericellamide D [8] & 3 & $\mathrm{H}$ & $\mathrm{Me}$ & $\mathrm{H}$ & emericellamide B [7] & 5 & $\mathrm{Me}$ & $\mathrm{Me}$ & $\mathrm{Me}$ \\
\hline
\end{tabular}

* Absolute configuration of C-3' and/or C-4' was not assigned.

\section{Materials and Methods}

\subsection{General Experimental Procedures}

Chemicals were purchased from Sigma-Aldrich or Merck unless otherwise specified. Analytical-grade solvents were used for solvent extractions. Solvents used for Sephadex chromatography, HPLC, UPLC, and HPLC-MS purposes were of HPLC grade supplied by Labscan or Sigma-Aldrich and filtered/degassed through $0.45 \mu \mathrm{m}$ polytetrafluoroethylene (PTFE) membrane prior to use. Deuterated solvents were purchased from Cambridge Isotopes (Tewksbury, MA, USA).

Microorganisms were manipulated under sterile conditions using a Laftech class II biological safety cabinet and incubated in either MMM Friocell incubators (Lomb Scientific, NSW, Australia) or an Innova 42R incubator shaker (John Morris, NSW, Australia) with temperature set at $26.5{ }^{\circ} \mathrm{C}$. Applikon micro-bioreactors were purchased from Enztech Pty Ltd. (Woollahra, NSW, Australia).

Liquid chromatography-diode array-mass spectrometry (HPLC-DAD-MS) data were acquired on an Agilent 1100 series separation module equipped with an Agilent 1100 series HPLC/MSD mass detector and diode array multiple wavelength detector. Semi-preparative and preparative 
HPLCs were performed using Agilent 1100 series HPLC instruments with corresponding detectors, fraction collectors and software inclusively. UPLC chromatograms were obtained on Agilent 1290 infinity UPLC system equipped with diode array multiple wavelength detector (Zorbax C8 RRHD $1.8 \mu \mathrm{m}$ column, $50 \times 2.1 \mathrm{~mm}$, eluting with $0.417 \mathrm{~mL} / \mathrm{min} 90 \% \mathrm{H}_{2} \mathrm{O} / \mathrm{MeCN}$ to $100 \% \mathrm{MeCN}$ (with isocratic $0.01 \%$ TFA modifier) over $2.50 \mathrm{~min}$ ). Ultra-high-performance-liquid-chromatography quadrupole-time-of-flight-mass-spectrometry (UHPLC-QTOF) analysis was performed on a UHPLC-QTOF instrument comprising an Agilent 1290 Infinity II UHPLC (Zorbax C 8 RRHD $1.8 \mu \mathrm{m}$ column, $50 \times 2.1 \mathrm{~mm}$, eluting with $0.5 \mathrm{~mL} / \mathrm{min}$ of isocratic $90 \% \mathrm{H}_{2} \mathrm{O} / \mathrm{MeCN}$ for $0.5 \mathrm{~min}$ followed by gradient elution to $100 \% \mathrm{MeCN}$ over $4.5 \mathrm{~min}$ (with isocratic $0.1 \%$ formic acid modifier) coupled to an Agilent 6545 Q-TOF. MS/MS analysis was performed on the same instrument for ions detected in the full scan at an intensity above 1000 counts at $10 \mathrm{scans} / \mathrm{s}$, with an isolation width of $4 \sim \mathrm{m} / \mathrm{z}$ using a fixed collision energy and a maximum of 3 selected precursors per cycle.

Chiroptical measurements $([\alpha] \mathrm{D})$ were obtained on a JASCO P-1010 polarimeter in a $100 \times 2 \mathrm{~mm}$ cell at specified temperatures. Nuclear magnetic resonance (NMR) spectra were acquired on a Bruker Avance $600 \mathrm{MHz}$ spectrometer with either a $5 \mathrm{~mm}$ PASEL 1H/D-13C Z-Gradient probe or $5 \mathrm{~mm}$ CPTCI 1H/19F-13C/15N/DZ-Gradient cryoprobe, controlled by TopSpin 2.1 software. In all cases spectra were acquired at $25^{\circ} \mathrm{C}$ in DMSO with referencing to residual ${ }^{1} \mathrm{H}$ or ${ }^{13} \mathrm{C}$ signals $\left(\delta_{\mathrm{H}} 2.50\right.$ and $\left.\delta_{\mathrm{C}} 39.51 \mathrm{ppm}\right)$ in the deuterated solvent, and in $\mathrm{MeOH}$ with referencing to residual ${ }^{1} \mathrm{H}$ or ${ }^{13} \mathrm{C}$ signals $\left(\delta_{\mathrm{H}} 3.31\right.$ and $\left.\delta_{\mathrm{C}} 49.15 \mathrm{ppm}\right)$ in the deuterated solvent. Electrospray ionization mass spectrometry (ESIMS) experiments were carried out on an Agilent 1100 series LC/MSD (quadrupole) instrument in both positive and negative modes. High-resolution ESIMS spectra were obtained on a Bruker micrOTOF mass spectrometer by direct injection in $\mathrm{MeOH}$ at $3 \mu \mathrm{L} / \mathrm{min}$ using sodium formate clusters as an internal calibrant.

\subsection{Fungal Strain Isolation and Taxonomy}

The fungal isolates CMB-F458, CMB-F585 and CMB-F115 were isolated from the gastrointestinal tract of a specimen of Mugil mullet fish, on M1 agar plates in presence of $3.3 \%$ artificial sea salt (M1S) incubated at $26.5^{\circ} \mathrm{C}$ for 8 days. Genomic DNA for the three fungi were extracted from their corresponding mycelia using the DNeasy Plant Mini Kit (Qiagen) as per the manufacturers protocol. The 18s rRNA genes were amplified by PCR using the universal internal transcribed spacer primers ITS-1 (5'-TCCGTAGGTGAACCTGCGG-3') and ITS-4 (5'-TCCTCCGCTTATTGATATGC-3') purchased from Sigma-Aldrich. The PCR mixture $(50 \mu \mathrm{L})$ contained $1 \mu \mathrm{L}$ of genomic DNA $(20-40 \mathrm{ng}), 200 \mu \mathrm{M}$ of each deoxynucleoside triphosphate (dNTP), $1.5 \mathrm{mM} \mathrm{MgCl}_{2}, 0.3 \mu \mathrm{M}$ of each primer, $1 \mathrm{U}$ of Taq DNA polymerase (Fisher Biotec) and $5 \mu \mathrm{L}$ of PCR buffer. PCR was performed using the following conditions: initial denaturation at $95^{\circ} \mathrm{C}$ for $3 \mathrm{~min}, 30$ cycles in series of $94{ }^{\circ} \mathrm{C}$ for $30 \mathrm{~s}$ (denaturation), $55^{\circ} \mathrm{C}$ for $60 \mathrm{~s}$ (annealing) and $72{ }^{\circ} \mathrm{C}$ for $60 \mathrm{~s}$ (extension), followed by one cycle at $72{ }^{\circ} \mathrm{C}$ for $6 \mathrm{~min}$. PCR products were purified with PCR purification kit (Qiagen, Victoria, Australia). Amplification products were examined by agarose gel electrophoresis. The DNA sequencing was performed by the Australian Genome Research Facility (AGRF) at the University of Queensland. BLAST analyses (NCBI database) on the ITS gene sequence for (a) CMB-F458 (GenBank accession no. MN080404) revealed 99\% identity with the fungal strain Scopulariopsis sp.; (b) CMB-F585 (GenBank accession no. MN080403) revealed 98\% identity with the fungal strain Beauveria sp.; and (c) CMB-F115 (GenBank accession no. MN080405) revealed $98 \%$ identity with the fungal strain Scopulariopsis sp.

\subsection{Scale up Cultivation of Scopulariopsis sp. CMB-F458}

A single colony of CMB-F458 was used to inoculate M1S agar plates $(\times 20)$, which were incubated at $26.5^{\circ} \mathrm{C}$ for 8 days, diced, extracted with EtOAc $(400 \mathrm{~mL})$, filtered and concentrated in vacuo at $40{ }^{\circ} \mathrm{C}$ to yield a crude extract $(80 \mathrm{mg}$ ). The crude extract was triturated (defatted) with $n$-hexane $(2 \times 10 \mathrm{~mL})$, after which the insolubles $(35 \mathrm{mg})$ were subjected to preparative reversed-phase HPLC (Phenomenex Luna- $\mathrm{C}_{18} 21.2 \mathrm{~mm} \times 25 \mathrm{~cm} \times 10 \mu \mathrm{m}$ column, $20 \mathrm{~mL} / \mathrm{min}$ gradient elution over $20 \mathrm{~min}$ 
from $90 \% \mathrm{H}_{2} \mathrm{O} / \mathrm{MeCN}$ to $100 \% \mathrm{MeCN}$ with a constant isocratic $0.01 \%$ TFA/MeCN) to yield scopularides A (1) (5 mg, 6.25\%) and B (2) (1.3 mg, 1.62\%) (Scheme S1).

\subsection{Scale-Up Cultivation of Beauveria sp. CMB-F585}

A single colony of CMB-F585 was used to inoculate YES agar plates $(\times 80)$, which were incubated at $26.5^{\circ} \mathrm{C}$ for 8 days, after which the agar was diced, extracted with EtOAc $(2 \times 500 \mathrm{~mL})$, filtered and concentrated in vacuo at $40{ }^{\circ} \mathrm{C}$ to yield a crude extract $(1.3 \mathrm{~g})$. The crude extract was sequentially triturated with $n$-hexane $(3 \times 50 \mathrm{~mL}), \mathrm{DCM}(3 \times 50 \mathrm{~mL})$ and $\mathrm{MeOH}(3 \times 50 \mathrm{~mL})$ to afford, after concentration in vacuo, $n$-hexane $(400 \mathrm{mg}), \mathrm{DCM}(800 \mathrm{mg})$ and $\mathrm{MeOH}(130 \mathrm{mg})$ solubles. A portion of the DCM solubles $\left(700 \mathrm{mg}\right.$ ) was subjected to gel chromatography (Sephadex ${ }^{\circledR} \mathrm{LH}-202.5 \times 70 \mathrm{~cm}$ column, gravity elution with isocratic $\mathrm{MeOH})$. Fractions $(10 \mathrm{~mL})$ were monitored by UPLC-QTOF analysis to reveal fraction -2-1 (200 $\mathrm{mg})$ as rich in target lipodepsipeptides, after which an aliquot $(60 \mathrm{mg})$ was subjected to semi-preparative reversed-phase HPLC (Zorbax Eclipse XDB-C $89.4 \mathrm{~mm} \times 25 \mathrm{~cm} \times 5 \mu \mathrm{m}$, $3 \mathrm{~mL} / \mathrm{min}$ isocratic elution over $40 \mathrm{~min}$ with $35 \% \mathrm{H}_{2} \mathrm{O} / \mathrm{MeCN}$ and $0.1 \% \mathrm{TFA} / \mathrm{MeCN}$ ) to yield scopularides C (3) (11.2 mg, 2.8\%), D (4) (5.5 mg, 1.4\%), E (5) (2.5 mg, 0.64\%), F (6) (1.7 mg, 0.43\%) and G (7) (0.3 mg, $0.07 \%$ ) (Scheme S2).

\subsection{Scale-Up Cultivation of Scopulariopsis sp. CMB-F115}

A single colony of CMB-F115 was used to inoculate YES agar plates $(\times 40)$ which were incubated at $26.5^{\circ} \mathrm{C}$ for 8 days, diced, extracted with EtOAc $(400 \mathrm{~mL} \times 2)$, filtered and concentrated in vacuo at $40{ }^{\circ} \mathrm{C}$, to yield a crude extract $(300 \mathrm{mg})$. The crude extract was sequentially triturated with $n$-hexane $(2 \times 50 \mathrm{~mL}), \mathrm{DCM}(2 \times 50 \mathrm{~mL})$ and $\mathrm{MeOH}(2 \times 50 \mathrm{~mL})$ to afford, after concentration in vacuo, $n$-hexane (60 mg), DCM (135 mg) and MeOH (65 mg) solubles. The DCM solubles, rich in target lipodepsipeptides, were subjected to gel chromatography (Sephadex ${ }^{\circledR}$ LH-20 $1.5 \times 22 \mathrm{~cm}$, isocratic gravity elution with $\mathrm{MeOH})$. Fractions $(10 \mathrm{~mL}$ ) were monitored by UPLC-QTOF analysis to reveal fraction -2-1 $(26 \mathrm{mg})$ as rich in target lipodepsipeptides, after which an aliquot $(20 \mathrm{mg})$ was subjected to semi-preparative reversed-phase HPLC (Zorbax Eclipse SB-C $39.4 \mathrm{~mm} \times 25 \mathrm{~cm} \times 5 \mu \mathrm{m}, 3 \mathrm{~mL} / \mathrm{min}$ isocratic elution over $32 \mathrm{~min}$ with $45 \% \mathrm{H}_{2} \mathrm{O} / \mathrm{MeCN}$ and $0.1 \% \mathrm{TFA} / \mathrm{MeCN}$ ) to yield scopularide $\mathrm{A}(\mathbf{1})(5 \mathrm{mg}, 2.1 \%)$ and $\mathrm{H}(\mathbf{8})$ ( $3.8 \mathrm{mg}, 1.6 \%$ ) (Scheme S3). (Note: Percentage yields estimated on a mass-to-mass basis against the weight of the crude EtOAc extract partition).

\subsection{Metabolite Charcterization}

Scopularide A (1); white powder; $[\alpha]_{\mathrm{D}}^{22.8}-34.9(c 0.25, \mathrm{MeOH})$; NMR $\left(600 \mathrm{MHz}, \mathrm{MeOH}-d_{4}\right)$ see Table S1, Figures S6 and S7; NMR (600 MHz, DMSO- $\left.d_{6}\right)$ see Table S2, Figures S8 and S9; ESI(+)MS $m / z 672[\mathrm{M}+\mathrm{H}]^{+} ; \mathrm{HRESI}(+) \mathrm{MS} m / z 694.4149[\mathrm{M}+\mathrm{Na}]^{+}$(calcd. for $\mathrm{C}_{36} \mathrm{H}_{57} \mathrm{~N}_{5} \mathrm{O}_{7} \mathrm{Na}$, 694.4150); GNPS library CCMSLIB00005436489.

Scopularide B (2); white powder; $[\alpha]_{\mathrm{D}}^{22.2}-19.8$ ( $c$ 0.05, MeOH); NMR (600 MHz, MeOH- $\left.d_{4}\right)$ see Table S3, Figures S10 and S11; NMR (600 MHz, DMSO- $\left.d_{6}\right)$ see Table S4, Figures S12 and S13; ESI(+)MS $m / z 644[\mathrm{M}+\mathrm{H}]^{+} ; \mathrm{HRESI}(+) \mathrm{MS} m / z 666.3842[\mathrm{M}+\mathrm{Na}]^{+}$(calcd. for $\mathrm{C}_{34} \mathrm{H}_{53} \mathrm{~N}_{5} \mathrm{O}_{7} \mathrm{Na}, 666.3837$ ); GNPS library CCMSLIB00005436490.

Scopularide C (3); white powder; $[\alpha]_{\mathrm{D}}^{22.2}-32.0$ ( $\left.c 0.13, \mathrm{MeOH}\right)$; NMR (600 MHz, DMSO- $\left.d_{6}\right)$ see Table 1 and Table S5, Figures S14-S18; ESI(+)MS $m / z 714[\mathrm{M}+\mathrm{H}]^{+}$; HRESI(+)MS $m / z 736.4638\left[\mathrm{M}+\mathrm{Na}^{+}\right.$ (calcd. for $\mathrm{C}_{39} \mathrm{H}_{63} \mathrm{~N}_{5} \mathrm{O}_{7} \mathrm{Na}$, 736.4620); GNPS library CCMSLIB00005436483.

Scopularide D (4); white powder; $[\alpha]_{\mathrm{D}}^{21.9}-40.9$ ( $\left.c 0.13, \mathrm{MeOH}\right)$; NMR (600 MHz, DMSO- $\left.d_{6}\right)$ see Table 1 and Table S6, Figures S19-S23; ESI(+)MS m/z $666[\mathrm{M}+\mathrm{H}]^{+}$; HRESI(+)MS m/z $688.4620\left[\mathrm{M}+\mathrm{Na}^{+}\right.$ (calcd. for $\mathrm{C}_{35} \mathrm{H}_{63} \mathrm{~N}_{5} \mathrm{O}_{7} \mathrm{Na}$, 688.4620); GNPS library CCMSLIB00005436484. 
Scopularide E (5); white powder; $[\alpha]_{\mathrm{D}}^{21.6}-19.2$ ( $c$ 0.13, MeOH); NMR (600 MHz, DMSO- $\left.d_{6}\right)$ see Table 1 and Table S7, Figures S24-S28; ESI(+)MS m/z $638[\mathrm{M}+\mathrm{H}]^{+}$; HRESI(+)MS m/z $660.4313[\mathrm{M}+\mathrm{Na}]^{+}$ (calcd. for $\mathrm{C}_{33} \mathrm{H}_{59} \mathrm{~N}_{5} \mathrm{O}_{7} \mathrm{Na}$, 660.4307); GNPS library CCMSLIB00005436485.

Scopularide F (6); white powder; $[\alpha]_{\mathrm{D}} 24.2-17.5$ ( $\left.0.1, \mathrm{MeOH}\right)$; NMR (600 MHz, DMSO- $\left.d_{6}\right)$ see Table 2 and Table S8, Figures S29-S33; ESI(+)MS $m / z 728[\mathrm{M}+\mathrm{H}]^{+}$; HRESI(+)MS m/z $750.4787\left[\mathrm{M}+\mathrm{Na}^{+}\right.$ (calcd. for $\mathrm{C}_{40} \mathrm{H}_{65} \mathrm{~N}_{5} \mathrm{O}_{7} \mathrm{Na}$, 750.4776); GNPS library CCMSLIB00005436486.

Scopularide G (7); white powder; ESI(+)MS m/z $680[\mathrm{M}+\mathrm{H}]^{+}$; HRESI(+)MS m/z 702.4789 [M + Na] ${ }^{+}$ (calcd. for $\mathrm{C}_{36} \mathrm{H}_{65} \mathrm{~N}_{5} \mathrm{O}_{7} \mathrm{Na}$, 702.4776) GNPS library CCMSLIB00005436487.

Scopularide H (8); white powder; $[\alpha]_{\mathrm{D}}^{22.2}-31.0$ ( 0 0.15, MeOH); NMR (600 MHz, DMSO- $d_{6}$ ) see Table 2 and Table S9, Figures S34-S38; ESI(+)MS m/z $700[\mathrm{M}+\mathrm{H}]^{+}$; HRESI(+)MS m/z $722.4464\left[\mathrm{M}+\mathrm{Na}^{+}\right.$ (calcd. for $\mathrm{C}_{38} \mathrm{H}_{61} \mathrm{~N}_{5} \mathrm{O}_{7} \mathrm{Na}$, 722.4463); GNPS library CCMSLIB00005436488.

\section{7. $C_{3}$ Marfey's Analyses}

Individual aliquots $(50 \mu \mathrm{g})$ of $1-8$ in $6 \mathrm{M} \mathrm{HCl}(100 \mu \mathrm{L})$ were heated to $100^{\circ} \mathrm{C}$ in sealed vials for 8 to $12 \mathrm{~h}$, after which the hydrolysates were concentrated to dryness at $40^{\circ} \mathrm{C}$ under a stream of dry $\mathrm{N}_{2}$. The hydrolysates were then treated with $1 \mathrm{M} \mathrm{NaHCO}_{3}(20 \mu \mathrm{L})$ and L-FDAA $(1 \%$ solution in acetone, $40 \mu \mathrm{L})$ at $40{ }^{\circ} \mathrm{C}$ for $1 \mathrm{~h}$, after which each reaction was neutralized with $1 \mathrm{M} \mathrm{HCl}(20 \mu \mathrm{L})$ and filtered $(0.45 \mu \mathrm{m}$ PTFE) prior to HPLC-DAD-ESIMS analysis. An aliquot $(10 \mu \mathrm{L})$ of each analyte was injected into an Agilent Zorbax SB- $\mathrm{C}_{3}$ column, $5 \mu \mathrm{m}, 150 \times 4.6 \mathrm{~mm}, 50{ }^{\circ} \mathrm{C}$, with a $1 \mathrm{~mL} / \mathrm{min}, 55 \mathrm{~min}$ linear gradient elution from $85 \%$ to $40 \% \mathrm{H}_{2} \mathrm{O} / \mathrm{MeOH}$ with a $5 \%$ isocratic modifier of $1 \%$ formic acid/MeCN. The analyte amino acid content was assessed by UV (340 $\mathrm{nm})$ and ESI $( \pm)$ MS monitoring, supported by SIE, with comparison to authentic standards. Amino acid standards were subjected to derivatization with L- and D-FDAA and HPLC-DAD-ESIMS analysis as described above for 1-8.

\subsection{Global Natural Product Social (GNPS) Molecular Networking}

A GNPS analysis of $\times 63$ Mugil mullet GIT-derived fungal isolates was carried out on UPLC-QTOF-(+)MS/MS data acquired on EtOAc crude extracts. Aliquots $(1 \mu \mathrm{L})$ of test solutions comprising $100 \mu \mathrm{g} / \mathrm{mL}$ of analyte in $100 \mu \mathrm{L} \mathrm{MeOH}$ were analysed on an Agilent 6545 Q-TOF LC/MS equipped with an Agilent 1290 infinity II UPLC system, utilising an Agilent SB- $\mathrm{C}_{8} 1.8 \mu \mathrm{m}, 2.1 \times 50 \mathrm{~mm}$ column, $0.5 \mathrm{~min}$ isocratic elution of $90 \% \mathrm{H}_{2} \mathrm{O} / \mathrm{MeCN}$ followed by $4.5 \mathrm{~min}$ gradient elution to $100 \%$ $\mathrm{MeCN}$ with a flow rate of $0.5 \mathrm{~mL} / \mathrm{min}$ and a constant isocratic $0.1 \%$ formic acid/MeCN modifier. UPLC-QTOF-(+)MS/MS data acquired for all samples at collision energy of $40 \mathrm{eV}$ were converted from Agilent MassHunter data files (.d) to mzXML file format using MSConvert software, and transferred to the GNPS server (gnps.ucsd.edu). Molecular networking was performed using the GNPS data analysis workflow using the spectral clustering algorithm with a cosine score of 0.7 and a minimum of 6 matched peaks. The resulting spectral network was imported into Cytoscape version 3.5.1, where nodes corresponding to media components and solvent were subtracted. Remaining nodes represented parent $(+) \mathrm{m} / \mathrm{z}$ of metabolites detected in analysed extracts, with node size indicating metabolite abundance and edge thickness corresponding to cosine scores (Figure 2, Figure 3, Figure 8, Figures S53 and S54). Figure S53 represents the molecular network for $\times 63$ GIT-derived fungal isolates, with the "scopularides" cluster represented in Figure 2. Similarly, scopularides molecular family clusters detected in CMB-F585, CMB-F115 and CMB-F458 crude extracts are represented in Figure 3, Figure 8 and Figure S54.

The data were deposited to the GNPS-MassIVE Datasets and are publicly accessible through the links listed below:

Fish microbial library molecular networking MASSIVE: MSV000084190

(https://massive.ucsd.edu/ProteoSAFe/dataset.jsp?task=479a543e9b45408bbe93414769058784). 
GNPS Molecular Networking jobs:

(https://gnps.ucsd.edu/ProteoSAFe/status.jsp?task=1abcf2c49b9a43cf85e19b8573eeb8a7)

(https://gnps.ucsd.edu/ProteoSAFe/status.jsp?task=2794a93e36724eaebafa0e1c3fb1aeb9)

(https://gnps.ucsd.edu/ProteoSAFe/status.jsp?task=430bfc8c34974e418156d8c6e711bd35).

CMB-F585 molecular networking

MASSIVE: MSV000084191

(https://massive.ucsd.edu/ProteoSAFe/dataset.jsp?task=4dee2595568c4296a1c4393de4758b24).

GNPS Molecular Networking job:

(https://gnps.ucsd.edu/ProteoSAFe/status.jsp?task=ae56648aefa54f789e04cfc314cb59fc).

CMB-F115 molecular networking

MASSIVE: MSV000084192

(https://massive.ucsd.edu/ProteoSAFe/dataset.jsp?task=74da14126ec24f2ca62ba443a05af7d2).

GNPS Molecular Networking job:

(https://gnps.ucsd.edu/ProteoSAFe/status.jsp?task=e90bdf9596ff4bbfb9e4eb200435a2b1).

CMB-F458 molecular networking

MASSIVE: MSV000084193

(https://massive.ucsd.edu/ProteoSAFe/dataset.jsp?task=4b0ec7ee53c34a299fe1828588ead84d).

GNPS Molecular Networking:

(https://gnps.ucsd.edu/ProteoSAFe/status.jsp?task=3c2af674f0a248c39149993d91a8149e)

\subsection{X-Ray Analysis of Scopularides C (3) and H (8)}

Single crystals of both 3 and 8 were obtained by slow evaporation from $20 \%$ aqueous $\mathrm{MeOH}$ at room temperature. Crystallographic data $\left(\mathrm{CuK} \alpha, 2 \theta_{\max }=125^{\circ}\right)$ were collected on an Oxford Diffraction Gemini S Ultra CCD diffractometer with the crystal cooled to $190 \mathrm{~K}$ with an Oxford Cryosystems Desktop Cooler. Data reduction and empirical absorption corrections were carried out with the CrysAlisPro program (Oxford Diffraction vers. 171.38.46). The structures were solved by direct methods with SHELXT and refined with SHELXL [21]. The thermal ellipsoid diagrams were generated with ORTEP3 [22], while Figures 7 and 9 were generated by Mercury 4.1.0. All crystallographic calculations were carried out within the WinGX graphical user interface [23]. The crystal structures have been deposited in the CCDC database (scoupularide C (3): CCDC 1936745 and scopularide H (8): CCDC 1936746) (Figure 7, Figure 9, Figures S55 and S56).

\subsection{Antibacterial Assay}

The bacterium to be tested was streaked onto a tryptic soy agar plate and was incubated at $37^{\circ} \mathrm{C}$ for $24 \mathrm{~h}$. One colony was then transferred to fresh tryptic soy broth $(15 \mathrm{~mL})$ and the cell density was adjusted to $10^{4}-10^{5} \mathrm{CFU} / \mathrm{mL}$. The compounds to be tested were dissolved in DMSO and diluted with $\mathrm{H}_{2} \mathrm{O}$ to give $600 \mu \mathrm{M}$ stock solution (20\% DMSO), which was serially diluted with $20 \%$ DMSO to give concentrations from 600 to $0.2 \mu \mathrm{M}$ in $20 \%$ DMSO. An aliquot $(10 \mu \mathrm{L})$ of each dilution was transferred to a 96-well microtiter plate and freshly prepared microbial broth $(190 \mu \mathrm{L})$ was added to each well to give final concentrations of 30 to $0.01 \mu \mathrm{M}$ in $1 \%$ DMSO. The plates were incubated at $37^{\circ} \mathrm{C}$ for $24 \mathrm{~h}$ and the optical density of each well was measured spectrophotometrically at $600 \mathrm{~nm}$ using POLARstar Omega plate (BMG LABTECH, Offenburg, Germany). Each test compound was screened against the Gram -ve bacteria Escherichia coli ATCC 11775 and the Gram +ve bacteria Staphylococcus aureus ATCC 9144 and three clinical isolates of methicillin-susceptible Staphylococcus aureus, methicillin-resistant Staphylococcus aureus and vancomycin-resistant Enterococcus faecalis. Rifampicin, ampicillin, daptomycin and methicillin were used as a positive control (30 $\mu \mathrm{M}$ in 10\% DMSO). The $\mathrm{IC}_{50}$ value was calculated 
as the concentration of the compound or antibiotic required for $50 \%$ inhibition of the bacterial cells using Prism 7.0 (GraphPad Software Inc., La Jolla, CA, USA).

\subsection{Antifungal Assay}

The fungus Candida albicans ATCC 10231 was streaked onto a Sabouraud agar plate and was incubated at $37^{\circ} \mathrm{C}$ for $48 \mathrm{~h}$. One colony was then transferred to fresh Sabouraud broth $(15 \mathrm{~mL})$ and the cell density adjusted to $10^{4}-10^{5} \mathrm{CFU} / \mathrm{mL}$. Test compounds were dissolved in DMSO and diluted with $\mathrm{H}_{2} \mathrm{O}$ to give a $600 \mu \mathrm{M}$ stock solution (20\% DMSO), which was serially diluted with $20 \%$ DMSO to give concentrations from 600 to $0.2 \mu \mathrm{M}$ in $20 \%$ DMSO. An aliquot $(10 \mu \mathrm{L})$ of each dilution was transferred to a 96-well microtiter plate and freshly prepared fungal broth $(190 \mu \mathrm{L})$ was added to each well to give final concentrations of 30 to $0.01 \mu \mathrm{M}$ in $1 \%$ DMSO. The plates were incubated at $37^{\circ} \mathrm{C}$ for $24 \mathrm{~h}$ and the optical density of each well was measured spectrophotometrically at $600 \mathrm{~nm}$ using POLARstar Omega plate (BMG LABTECH, Offenburg, Germany). Ketoconazole was used as a positive control $(30 \mu \mathrm{g} / \mathrm{mL}$ in $10 \% \mathrm{DMSO})$. Where relevant, $\mathrm{IC}_{50}$ value were calculated as the concentration of the compound or antifungal drug required for 50\% inhibition of the fungal cells using Prism 7.0 (GraphPad Software Inc., La Jolla, CA, USA).

\subsection{Cytotoxicity Assay}

Adherent SW620 (human colorectal carcinoma) and NCI-H460 (human lung carcinoma) cells were cultured in Roswell Park Memorial Institute (RPMI) 1640 medium, while HepG2 (adherent human hepatocellular carcinoma) cells were cultured in Dulbecco's modified Eagle's medium (DMEM). All cells were cultured as adherent mono-layers in flasks supplemented with $10 \%$ foetal bovine serum, L-glutamine $(2 \mathrm{mM})$, penicillin $(100 \mathrm{unit} / \mathrm{mL})$ and streptomycin $(100 \mu \mathrm{g} / \mathrm{mL})$, in a humidified $37^{\circ} \mathrm{C}$ incubator supplied with $5 \% \mathrm{CO}_{2}$. Briefly, cells were harvested with trypsin and dispensed into 96-well microtiter assay plates at 3000 cells/well, after which they were incubated for $18 \mathrm{~h}$ at $37^{\circ} \mathrm{C}$ with $5 \% \mathrm{CO}_{2}$ (to allow cells to attach as adherent mono-layers). Test compounds were dissolved in $20 \%$ DMSO in PBS $(v / v)$ and aliquots $(10 \mu \mathrm{L})$ applied to cells over a series of final concentrations ranging from $10 \mathrm{nM}$ to $30 \mu \mathrm{M}$. After $48 \mathrm{~h}$ incubation at $37^{\circ} \mathrm{C}$ with $5 \% \mathrm{CO}_{2}$, an aliquot $(10 \mu \mathrm{L})$ of 3-(4,5-dimethylthiazol-2-yl)-2,5-diphenyltetrazolium bromide (MTT) in phosphate buffered saline (PBS, $5 \mathrm{mg} / \mathrm{mL}$ ) was added to each well (final concentration $0.5 \mathrm{mg} / \mathrm{mL}$ ), and microtiter plates were incubated for a further $4 \mathrm{~h}$ at $37^{\circ} \mathrm{C}$ with $5 \% \mathrm{CO}_{2}$. After final incubation, the medium was aspirated and precipitated formazan crystals dissolved in DMSO (100 $\mu \mathrm{L} /$ well). The absorbance of each well was measured at $600 \mathrm{~nm}$ with a POLARstar Omega plate (BMG LABTECH, Offenburg, Germany). Where relevant, $\mathrm{IC}_{50}$ values were calculated using Prism 8.0, as the concentration of analyte required for $50 \%$ inhibition of cancer cell growth (compared to negative controls). Negative control was $1 \%$ aqueous DMSO, while positive control was vinblastine $(30 \mu \mathrm{M})$. All experiments were performed in duplicate.

Supplementary Materials: The following are available online at http://www.mdpi.com/1660-3397/17/8/475/s1, MS/MS fragmentations, Marfey's analyses, together with tabulated 1D and 2D NMR data, annotated 1D NMR and selected 2D NMR spectra, and bioassay data.

Author Contributions: R.J.C. conceptualized the research; A.H.E. carried out the isolation and spectroscopic characterization of isolated compounds, and performed Marfey's and GPNS analyses; A.H.E. and Z.G.K. performed the taxonomic identification of the fungal strains; Z.G.K. performed the bioassays; P.V.B. performed the X-ray analyses; A.H.E. and Z.G.K. assigned molecular structures, and constructed the Supplementary Materials document; R.J.C. reviewed all data and drafted the manuscript, with support from A.H.E. and Z.G.K.

Funding: This work was funded in part by the Institute for Molecular Bioscience, and the University of Queensland.

Acknowledgments: The authors thank P. Abdala for assistance in fungal isolations. AHE acknowledges the provision of a University of Queensland, International Postgraduate Scholarship.

Conflicts of Interest: The authors declare no conflict of interest. 


\section{References}

1. Mohamed, O.G.; Khalil, Z.G.; Capon, R.J. Prolinimines: N-Amino-L-Pro-methyl ester (hydrazine) Schiff bases from a fish gastrointestinal tract-derived fungus, Trichoderma sp. CMB-F563. Org. Lett. 2018, 20, 377-380. [CrossRef] [PubMed]

2. Yu, Z.; Lang, G.; Kajahn, I.; Schmaljohann, R.; Imhoff, J.F. Scopularides A and B, cyclodepsipeptides from a marine sponge-derived fungus, Scopulariopsis brevicaulis. J. Nat. Prod. 2008, 71, 1052-1054. [CrossRef] [PubMed]

3. Wang, M.; Carver, J.J.; Phelan, V.V.; Sanchez, L.M.; Garg, N.; Peng, Y.; Nguyen, D.D.; Watrous, J.; Kapono, C.A.; Luzzatto-Knaan, T.; et al. Sharing and community curation of mass spectrometry data with Global Natural Products Social Molecular Networking. Nat. Biotechnol. 2016, 34, 828-837. [CrossRef] [PubMed]

4. Vijayasarathy, S.; Prasad, P.; Fremlin, L.J.; Ratnayake, R.; Salim, A.A.; Khalil, Z.; Capon, R.J. C 3 and 2D C 3 Marfey's methods for amino acid analysis in natural products. J. Nat. Prod. 2016, 79, 421-427. [CrossRef] [PubMed]

5. Ding, L.-J.; Yuan, W.; Liao, X.-J.; Han, B.-N.; Wang, S.-P.; Li, Z.-Y.; Xu, S.-H.; Zhang, W.; Lin, H.-W. Oryzamides A-E, cyclodepsipeptides from the sponge-derived fungus Nigrospora oryzae PF18. J. Nat. Prod. 2016, 79, 2045-2052. [CrossRef] [PubMed]

6. Hou, X.-M.; Li, Y.-Y.; Shi, Y.-W.; Fang, Y.W.; Chao, R.; Gu, Y.-C.; Wang, C.-Y.; Shao, C.-L. Integrating molecular networking and ${ }^{1} \mathrm{H}$ NMR to target the isolation of chrysogeamides from a library of marine-derived Penicillium fungi. J. Org. Chem. 2019, 84, 1228-1237. [CrossRef] [PubMed]

7. Oh, D.-C.; Kauffman, C.A.; Jensen, P.R.; Fenical, W. Induced production of emericellamides A and B from the marine-derived fungus Emericella sp. in competing co-culture. J. Nat. Prod. 2007, 70, 515-520. [CrossRef]

8. Chiang, Y.-M.; Szewczyk, E.; Nayak, T.; Davidson, A.D.; Sanchez, J.F.; Lo, H.-C.; Ho, W.-Y.; Simityan, H.; Kuo, E.; Praseuth, A.; et al. Molecular genetic mining of the Aspergillus secondary metabolome: Discovery of the emericellamide biosynthetic pathway. Chem. Biol. 2008, 15, 527-532. [CrossRef]

9. Wu, H.M.; Lin, L.P.; Xu, Q.L.; Han, W.B.; Zhang, S.; Liu, Z.W.; Mei, Y.N.; Yao, Z.-J.; Tan, R.X. Nodupetide, a potent insecticide and antimicrobial from Nodulisporium sp. associated with Riptortus pedestris. Tetrahedron Letts. 2017, 58, 663-665. [CrossRef]

10. Vining, L.C.; Taber, W.A. Isariin, a new depsipeptide from Isaria cretacea. Can. J. Chem. 1962, 40, $1579-1584$. [CrossRef]

11. Ióca, L.P.; Nicacio, K.J.; Berlinck, R.G.S.; Ióca, L.P.; Nicacio, K.J.; Berlinck, R.G.S. Natural Products from marine invertebrates and microorganisms in Brazil between 2004 and 2017: Still the challenges, more rewards. J. Braz. Chem. Soc. 2018, 29, 998-1031. [CrossRef]

12. Lira, S.P.; Vita-marques, A.M.; Seleghim, M.H.R.; Bugni, T.S.; Labarbera, D.V.; Sette, L.D.; Sponchiado, S.R.P.; Ireland, C.M.; Berlinck, R.G.S. New destruxins from the marine-derived fungus Beauveria felina. J. Antibiot. 2006, 59, 553-563. [CrossRef] [PubMed]

13. Baute, R.; Deffieux, G.; Merlet, D.; Baute, M.A.; Neveu, A. New insecticidal cyclodepsipeptides from the fungus Isaria felina: I. Production, isolation and insecticidal properties of isariins B, C and D. J. Antibiot. 1981, 34, 1261-1265. [CrossRef] [PubMed]

14. Deffieux, G.; Merlet, D.; Baute, R.; Bourgeois, G.; Baute, M.A.; Neveu, A. New insecticidal cyclodepsipeptides from the fungus Isaria felina II. Structure elucidation of isariins B, C and D. J. Antibiot. 1981, 34, 1266-1270. [CrossRef] [PubMed]

15. Sabareesh, V.; Ranganayaki, R.S.; Raghothama, S.; Bopanna, M.P.; Balaram, H.; Srinivasan, M.C.; Balaram, P. Identification and characterization of a library of microheterogeneous cyclohexadepsipeptides from the fungus Isaria. J. Nat. Prod. 2007, 70, 715-729. [CrossRef] [PubMed]

16. Langenfeld, A.; Blond, A.; Gueye, S.; Herson, P.; Nay, B.; Dupont, J.; Prado, S. Insecticidal cyclodepsipeptides from Beauveria felina. J. Nat. Prod. 2011, 74, 825-830. [CrossRef] [PubMed]

17. Du, F.-Y.; Li, X.-M.; Zhang, P.; Li, C.-S.; Wang, B.-G. Cyclodepsipeptides and other O-containing heterocyclic metabolites from Beauveria felina EN-135, a marine-derived entomopathogenic fungus. Mar. Drugs 2014, 12, 2816-2826. [CrossRef] [PubMed]

18. Asolkar, R.N.; Freel, K.C.; Jensen, P.R.; Fenical, W.; Kondratyuk, T.P.; Park, E.-J.; Pezzuto, J.M. Arenamides A-C, cytotoxic NFkappaB inhibitors from the marine actinomycete Salinispora arenicola. J. Nat. Prod. 2009, 72, 396-402. [CrossRef] 
19. Hardy, P.M.; Prout, R.A.; Rydon, H.N. Polypeptides. Part XXV. Synthesis of isariin. J. Chem. Soc. Perkin Trans. 1 1974, 802-808. [CrossRef]

20. Chandrasekhar, S.; Pavankumarreddy, G.; Sathish, K. Total synthesis of arenamide A and its diastereomer. Tetrahedron Letts. 2009, 50, 6851-6854. [CrossRef]

21. Sheldrick, G.M. A short history of SHELX. Acta Crystallogr. Sect. A Found. Crystallogr. 2008, 64, 112-122. [CrossRef] [PubMed]

22. Farrugia, L.J. ORTEP-3 for Windows-A version of ORTEP-III with a Graphical User Interface (GUI). J. Appl. Crystallogr. 1997, 30, 565. [CrossRef]

23. Farrugia, L.J. WinGX suite for small-molecule single-crystal crystallography. J. Appl. Crystallogr. 1999, 32, 837-838. [CrossRef]

(C) 2019 by the authors. Licensee MDPI, Basel, Switzerland. This article is an open access article distributed under the terms and conditions of the Creative Commons Attribution (CC BY) license (http://creativecommons.org/licenses/by/4.0/). 